\title{
LIMIT THEOREMS FOR HITTING TIMES OF 1-DIMENSIONAL GENERALIZED DIFFUSIONS
}

\author{
MATSUYO TOMISAKI AND MAKOTO YAMAZATO
}

\begin{abstract}
Limit theorems are obtained for suitably normalized hitting times of single points for 1-dimensional generalized diffusion processes as the hitting points tend to boundaries under an assumption which is slightly stronger than that the existence of limits $\gamma+1$ of the ratio of the mean and the variance of the hitting time. Laplace transforms of limit distributions are modifications of Bessel functions. Results are classified by the one parameter $\{\gamma\}$, each of which is the degree of corresponding Bessel function. In case the limit distribution is degenerate to one point, by changing the normalization, we obtain convergence to the normal distribution. Regarding the starting point as a time parameter, we obtain convergence in finite dimensional distributions to self-similar processes with independent increments under slightly stronger assumption.
\end{abstract}

\section{$\S 1$. Introduction}

We denote by $\mathcal{M}$ the class of right continuous non-decreasing functions $m:[-\infty, \infty] \rightarrow[-\infty, \infty]$, satisfying $m(-\infty)=-\infty, m(\infty)=\infty, m(0-)=$ 0 . For $m \in \mathcal{M}$, we set

$$
\begin{aligned}
& l_{1}(m)=\sup \{x<0 ; m(x)=-\infty\} \\
& l_{2}(m)=\inf \{x>0 ; m(x)=\infty\}
\end{aligned}
$$

If there is no confusion, we write $l_{i}(m)$ simply $l_{i}$ for $i=1,2$. We denote by $E_{m}$ the support of the measure induced by $m$ restricted to $\left(l_{1}, l_{2}\right)$. There naturally corresponds a strong Markov process $\left\{X_{t}\right\}$ (called 1-dimensional generalized diffusion process) on $E_{m}$ (whose formal infinitesimal generator is $\left.\frac{d}{d m} \frac{d}{d x}\right)$ to $m$ by changing time of the Brownian motion. The measure $m(d x)$ is called the speed measure of $\left\{X_{t}\right\}$. Denote the hitting time of $x$ for $\left\{X_{t}\right\}$ by $\tau_{x}$. We are concerned with a problem what is the suitable normalization and what is the limit distribution of $\tau_{x}$ when the process starts at the origin and $x$ tends to $l_{2}$.

Received June 6, 1996. 
The problem of the asymptotics of $\tau_{x}$ as $x \rightarrow l_{2}$ is closely related to metal exhaustion (private communication with A. Shimizu). That is, regarding the extreme point of a crack of metal by exhaustion as a maximal point of diffusion process, one is concerned with determining the durable length of time, by investigating asymptotics of the maximal point.

Keller-Kersting-Roesler [5] studied the problem in the case that the process is regular diffusion and the starting point (the origin) is a reflecting boundary. They showed that if the mean $E_{0} \tau_{x}$ and the variance $V_{0} \tau_{x}$ are finite and $\left(V_{0} \tau_{x}\right)^{1 / 2}=O\left(E_{0} \tau_{x}\right)($ as $x \rightarrow \infty)$, then a suitable normalization is

$$
\tilde{\tau}_{x}=\left(\tau_{x}-E_{0} \tau_{x}\right) /\left(V_{0} \tau_{x}\right)^{1 / 2}
$$

and, they obtained a necessary and sufficient condition for the limit distribution of $\tilde{\tau}_{x}$ to be normal or exponential. Moreover, they obtained sufficient conditions in terms of infinitesimal generator (diffusion coefficient and drift coefficient) that the limit distribution is to be normal or exponential.

Soloviev [9] studied the same problem for a birth and death process which starts at its boundary. His result is described in a unified form and is deeper than the result in [5].

In this paper, we consider the class of 1-dimensional generalized diffusion processes, which includes both birth and death processes and regular diffusion processes. We do not assume the starting point to be a reflecting boundary. The starting point can be other kind of bundary or an interior point. Our results are extensions of Keller-Kersting-Roesler and Soloviev even in the case that the starting point is a reflecting boundary. We remark that the class of hitting time distributions of 1-dimensional generalized diffusion processes is determined by [11] and [12]; it is a subclass of the class of infinitely divisible distributions.

We will give the conditions for convergence in terms of asymptotics of $m$ near $l_{2}$. Our results are classified by one parameter $0 \leqq \gamma \leqq \infty$, which appears in the condition

$$
m(x) \int_{0}^{x} m(y) d y / \int_{0}^{x} m(y)^{2} d y \longrightarrow 1+\gamma \quad \text { as } \quad x \rightarrow l_{2},
$$

or in some related conditions.

We describe, in Section 4, our result in case that the origin is a reflecting boundary. In Section 5, we describe the results in case that the origin is not a reflecting boundary. We can regard the hitting point $x$ as a time parameter 
and consider the convergence of $\left\{\tau_{x}\right\}$ in finite dimensional distributions of stochastic processes. Then we observe that the behavior of convergence is quite different according as $\gamma<1, \gamma=1$ and $\gamma>1$. In case $\gamma=\infty$, the limit distribution is degenerate to one point. We note in Section 6 that in this case, normal distribution appears as a limit distribution if we change the normalization. Section 7 is devoted to the comparison of our results with Keller-Kersting-Roesler's.

\section{$\S 2$. Hitting time distributions of 1-dimensional generalized diffu- sions}

Let $\{B(t)\}$ be 1-dimensional Brownian motion on a probability space $\left(\Omega, \mathcal{F}, \mathcal{F}_{t}, \theta_{t}, P_{x}\right)$ with $B(0)=0$, where $\mathcal{F}_{t}$ is an increasing right continuous family of sub $\sigma$-fields of $\mathcal{F}$ and $\theta_{t}$ is a shift operator. Let $l(t, x)$ be its local time. For $m \in \mathcal{M}$, set

$$
\phi(t)=\int_{\mathbf{R}=(-\infty, \infty)} l(t, x) d m(x) .
$$

This $\phi(t)$ is a non-decreasing function. Let $\phi^{-1}(t)$ be the right continuous inverse of $\phi(t)$. Let $E_{m}=\operatorname{supp}\left(\left.m\right|_{\left(l_{1}, l_{2}\right)}\right)$. For $t_{1}<t_{2}$,

$$
\phi\left(t_{2}\right)=\phi\left(t_{1}\right) \text { if and only if } B(t) \notin E_{m} \text { for } t \in\left(t_{1}, t_{2}\right) \text {. }
$$

Let $\zeta=\inf \left\{t \geqq 0: B\left(\phi^{-1}(t)\right)=l_{1}\right.$ or $\left.l_{2}\right\}$. By $(2.1)$, for any $t<\zeta, \phi^{-1}(t)$ is an increasing point of $\phi$. So, for $t$ with $t<\zeta, B\left(\phi^{-1}(t)\right) \in E_{m}$. Setting

$$
X_{t}=B\left(\phi^{-1}(t)\right), \quad \tilde{\mathcal{F}}_{t}=\mathcal{F}_{\phi^{-1}(t)}, \quad \tilde{\theta}_{t}=\theta_{\phi^{-1}(t)},
$$

we have that

$$
X=\left(\Omega, \mathcal{F}, \tilde{\mathcal{F}}_{t}, X_{t}, \tilde{\theta}_{t}, P_{x}\right)
$$

is a strong Markov process on $E_{m}$ and in case that the derivative $m^{\prime}$ of $m$ exists, the infinitesimal generator of $X$ is given by $\frac{1}{m^{\prime}(x)} \cdot \frac{d^{2}}{d x^{2}}$. Above $\zeta$ is the life time of $X_{t}$. We can show that if $B\left(\phi^{-1}(t)\right)=y$, then for each $x \in E_{m}$ satisfying $0<x<y$, there is $s<t$ such that $B\left(\phi^{-1}(s)\right)=x$. Let $\tau_{x}$ be the hitting time of $x$ for $X_{t}$. Then, $\tau_{y}(\omega)=t$ if and only if

$$
\tau_{x}(\omega)+\tau_{y}\left(\tilde{\theta}_{\tau_{x}} \omega\right)=t
$$


If $P_{0}\left(\tau_{x}<\infty\right)=1$ for $0<x<l_{2}$, then by the strong Markov property of $X$,

$$
\begin{aligned}
& E_{0}\left(e^{-\lambda\left(\tau_{y}(\omega)-\tau_{x}(\omega)\right)}\right) \\
= & E_{0}\left(E_{0}\left(e^{-\lambda \tau_{y}\left(\tilde{\theta}_{\tau_{x}} \omega\right)} \mid \mathcal{F}_{\tau_{x}}\right)\right) \\
= & E_{0}\left(E_{X_{\tau_{x}}}\left(e^{-\lambda \tau_{y}}\right)\right)=E_{x}\left(e^{-\lambda \tau_{y}}\right)
\end{aligned}
$$

and

$$
\begin{aligned}
& E_{0}\left(e^{-\lambda_{1} \tau_{x}(\omega)-\lambda_{2}\left(\tau_{y}(\omega)-\tau_{x}(\omega)\right)}\right) \\
= & E_{0}\left(e^{-\lambda_{1} \tau_{x}(\omega)} E_{0}\left(e^{-\lambda_{2}\left(\tau_{y}(\omega)-\tau_{x}(\omega)\right)} \mid \mathcal{F}_{\tau_{x}}\right)\right) \\
= & E_{0}\left(e^{-\lambda_{1} \tau_{x}(\omega)}\right) E_{x}\left(e^{-\lambda_{2} \tau_{y}}\right) \\
= & E_{0}\left(e^{-\lambda_{1} \tau_{x}(\omega)}\right) E_{0}\left(e^{-\lambda_{2}\left(\tau_{y}(\omega)-\tau_{x}(\omega)\right)}\right) .
\end{aligned}
$$

Therefore, if $P_{0}\left(\tau_{x}<\infty\right)=1$ for $0<x<l_{2}$, then $\left\{\tau_{x}\right\}$ has independent increments.

For $m \in \mathcal{M}$, let $\varphi(x, \lambda)$ and $\phi(x, \lambda)$ be the continuous solutions of

$$
\begin{aligned}
\varphi(x, \lambda) & =1+\lambda \int_{[0, x)}(x-y) \varphi(y, \lambda) d m(y) \text { for } x \geqq 0 \\
& =1-\lambda \int_{[x, 0)}(x-y) \varphi(y, \lambda) d m(y) \text { for } x<0
\end{aligned}
$$

and

$$
\begin{aligned}
\phi(x, \lambda) & =x+\lambda \int_{[0, x)}(x-y) \phi(y, \lambda) d m(y) \text { for } x \geqq 0 \\
& =x-\lambda \int_{[x, 0)}(x-y) \phi(y, \lambda) d m(y) \text { for } x<0
\end{aligned}
$$

respectively. Both are entire functions of $\lambda$. Since $\frac{d^{+}}{d x}\left(\frac{\phi(x, \lambda)}{\varphi(x, \lambda)}\right)=\frac{1}{\varphi(x, \lambda)^{2}}$, $\lim _{x \rightarrow l_{1}}-\phi(x, \lambda) / \varphi(x, \lambda)$ exists and we denote this limit by $h(\lambda)$. Here we denoted right derivative by $\frac{d^{+}}{d x}$. Relations between $h$ and $m$ restricted to $[-\infty, 0)$ is studied deeply by Kac and Krein (see [6]). Let

$$
u(x, \lambda)=\varphi(x, \lambda)+h(\lambda)^{-1} \phi(x, \lambda)
$$

Then, for $\lambda>0, u$ is a positive increasing function of $x$, satisfying either $u\left(l_{1}+, \lambda\right)=0$ or $\frac{d^{+}}{d x} u((-\infty)+, \lambda)=0$ according as $l_{1}>-\infty$ or $l_{1}=-\infty$. 
So, by the general theory on diffusion processes, the Laplace transform of $\tau_{x}$ is given by the following:

$$
E_{\xi}\left(e^{-\lambda \tau_{x}}\right)=u(\xi, \lambda) / u(x, \lambda) \quad \text { if } \quad l_{1}<\xi \leqq x<l_{2} .
$$

See Ito-McKean [3] p.128-129.

In the case $m \equiv 0$ on $(-\infty, 0), l_{1}=-\infty$, the origin is a reflecting boundary. Then, since $-\phi(x, \lambda) / \varphi(x, \lambda)=|x| / 1 \rightarrow \infty$ as $x \rightarrow-\infty$,

$$
E_{\xi}\left(e^{-\lambda \tau_{x}}\right)=\varphi(\xi, \lambda) / \varphi(x, \lambda), \quad-\infty<\xi \leqq x<l_{2}
$$

Note that $\left\{\tau_{x}\right\}$ is defined only for $x \in E_{m}$. By the independent increments property, the finite dimensional distribution of $\left\{\tau_{x}\right\}$ is given by

$$
\begin{aligned}
& E_{x_{0}}\left[\exp \left\{-\lambda_{1} \tau_{x_{1}}-\lambda_{2} \tau_{x_{2}}-\cdots-\lambda_{n} \tau_{x_{n}}\right\}\right] \\
= & \prod_{j=1}^{n} u\left(x_{j-1}, \sum_{k=j}^{n} \lambda_{k}\right) / u\left(x_{j}, \sum_{k=j}^{n} \lambda_{k}\right)
\end{aligned}
$$

for $x_{0}, x_{1}, \ldots, x_{n} \in E_{m}, x_{0}<x_{1}<\cdots<x_{n}$. Note that the finite dimensional distributions are determined only by $u(x, \lambda), x \in E_{m}$.

LEMMA 1. $u(s, \lambda) / u(t, \lambda)$ for $l_{1}<s \leqq t<l_{2}$ is the Laplace transform of a distribution.

Proof. We may assume that $s, t \notin E_{m}$. Let $m_{c}(d x)=m(d x)+c \delta_{s}(d x)+$ $c \delta_{t}(d x)$, where $c>0$ and $\delta_{a}(d x)$ is the delta distribution concentrated at $a$. Write $u$ corresponding to $m_{c}$ as $u_{c}$. Then, since $s, t \in E_{m_{c}}, u_{c}(s, \lambda) / u_{c}(t, \lambda)$ is the Laplace transform of a distribution. Since

$$
u_{c}(s, \lambda)=u(s, \lambda)
$$

and

$$
u_{c}(t, \lambda)=\varphi(t, \lambda)+(t-s) \lambda c \varphi(s, \lambda)+\{\phi(t, \lambda)+(t-s) \lambda c \phi(s, \lambda)\} / h(\lambda)
$$

we have

$$
u_{c}(s, \lambda) / u_{c}(t, \lambda) \rightarrow u(s, \lambda) / u(t, \lambda) \quad \text { as } \quad c \rightarrow 0,
$$

uniformly in any compact set of $\lambda$. Hence $u(s, \lambda) / u(t, \lambda)$ is the Laplace transform of a distribution. 
The above discussion leads to the conclusion that if we define finite dimensional distributions of $\left\{\tau_{x}\right\}$ by $(2.5)$ for $l_{1}<x_{0}<x_{1}<\cdots<x_{n}<l_{2}$, then they satisfy the consistency condition and therefore by Kolmogorov's extension theorem, there is a unique probability measure on $\mathbf{R}^{\left[x_{0}, l_{2}\right]}$ whose finite dimensional distributions coincide with (2.5). We call this stochastic process generalized hitting time process with starting point $x_{0}$.

$\S$ 3. Functions $\varphi, \phi$ and $h$ corresponding to special $m \in \mathcal{M}$

We define a function $\Phi_{\gamma}(\lambda),(0<\gamma<\infty)$ by

$$
\begin{aligned}
\Phi_{\gamma}(\lambda) & =\Gamma(\gamma) \lambda^{(1-\gamma) / 2} I_{\gamma-1}\left(2 \lambda^{1 / 2}\right) \\
& =\Gamma(\gamma) \sum_{n=0}^{\infty} \frac{\lambda^{n}}{n ! \Gamma(n+\gamma)}, \quad 0 \leqq \lambda<\infty
\end{aligned}
$$

where $I_{\nu}(z)$ is the modified Bessel function:

$$
I_{\nu}(z)=\left(\frac{z}{2}\right)^{\nu} \sum_{n=0}^{\infty} \frac{(z / 2)^{2 n}}{n ! \Gamma(\nu+n+1)} .
$$

Since $\lim _{\gamma \rightarrow 0} \Phi_{\gamma}(\gamma \lambda)=1+\lambda$ and $\lim _{\gamma \rightarrow \infty} \Phi_{\gamma}(\gamma \lambda)=e^{\lambda}$, we define

$$
\begin{gathered}
\left.\Phi_{\gamma}(\gamma \lambda)\right|_{\gamma=0}=1+\lambda \\
\left.\Phi_{\gamma}(\gamma \lambda)\right|_{\gamma=\infty}=e^{\lambda} .
\end{gathered}
$$

EXAMPLE 1. Let $c_{1}, c_{2} \geqq 0, l_{1}<-a_{1} \leqq 0 \leqq a_{2}<l_{2}$ and

$$
m(\xi)=\left\{\begin{array}{cl}
-c_{1} & \text { for } \quad l_{1}<\xi<-a_{1} \\
0 & \text { for } \quad-a_{1} \leqq \xi<a_{2} \\
c_{2} & \text { for } \quad a_{2} \leqq \xi<l_{2}
\end{array}\right.
$$

Then, the corresponding solutions $\varphi, \phi$ of (2.2) and (2.3) and $h$ function are

$$
\begin{gathered}
\varphi(x, \lambda)= \begin{cases}1-c_{1}\left(x+a_{1}\right) \lambda & \text { for } l_{1}<x \leqq-a_{1}, \\
1 & \text { for }-a_{1}<x \leqq a_{2}, \\
1+c_{2}\left(x-a_{2}\right) \lambda & \text { for } a_{2}<x<l_{2},\end{cases} \\
\phi(x, \lambda)= \begin{cases}x+c_{1} a_{1}\left(x+a_{1}\right) \lambda & \text { for } l_{1}<x \leqq-a_{1}, \\
x & \text { for }-a_{1}<x \leqq a_{2}, \\
x+c_{2} a_{2}\left(x-a_{2}\right) \lambda & \text { for } a_{2}<x<l_{2},\end{cases}
\end{gathered}
$$


and

$$
h(\lambda)= \begin{cases}\left(l_{1}+c_{1} a_{1}\left(l_{1}+a_{1}\right) \lambda\right) /\left(-1+c_{1}\left(l_{1}+a_{1}\right) \lambda\right) \\ & \text { if } c_{1}>0 \text { and } l_{1}>-\infty \\ \left(1+c_{1} a_{1} \lambda\right) / c_{1} \lambda & \text { if } c_{1}>0 \text { and } l_{1}=-\infty \\ -l_{1} & \text { if } c_{1}=0\end{cases}
$$

respectively.

EXAMPLE 2. Let $\left|l_{i}\right|=\infty, i=1,2,0<\gamma<1, c_{1}, c_{2} \geqq 0$ and

$$
m(x)= \begin{cases}-c_{1}|x|^{\gamma /(1-\gamma)} /(1-\gamma) & \text { for } x<0 \\ -c_{2} x^{\gamma /(1-\gamma)} /(1-\gamma) & \text { for } x \geqq 0\end{cases}
$$

We have

$$
\varphi(x, \lambda)= \begin{cases}\Phi_{\gamma}\left(c_{1}|x|^{1 /(1-\gamma)} \gamma \lambda\right) & \text { for } x<0 \\ \Phi_{\gamma}\left(c_{2} x^{1 /(1-\gamma)} \gamma \lambda\right) & \text { for } x \geqq 0 .\end{cases}
$$

Also we have

$$
\phi(x, \lambda)= \begin{cases}x \phi_{2-\gamma}\left(c_{1}|x|^{1 /(1-\gamma)} \gamma \lambda\right) & \text { for } x<0 \\ x \phi_{2-\gamma}\left(c_{2} x^{1 /(1-\gamma)} \gamma \lambda\right) & \text { for } x \geqq 0\end{cases}
$$

Moreover, we have

$$
h(\lambda)=E_{\gamma}\left(c_{1} \lambda\right)^{\gamma-1}, \quad \lambda>0
$$

where

$$
E_{\gamma}=\gamma^{\gamma-1} \Gamma(2-\gamma) / \Gamma(\gamma)
$$

Obviously, if $c_{1}=0$, then $\varphi(x, \lambda)=1$ and $\phi(x, \lambda)=x$ for $x<0$. Since $E_{\gamma} \rightarrow 1$ as $\gamma \downarrow 0$, Example 1 with $c_{1}=a_{2}=0$ can be regarded as a special case $\gamma=0, c_{1}=0$, of this example by (3.2).

EXAMPLE 3. Let, for $\gamma>1$,

$$
m(x)=|x|^{\gamma /(1-\gamma)} /(\gamma-1), \quad x<0 .
$$

Then

$$
u(x, \lambda)=\Phi_{\gamma}\left(|x|^{1 /(1-\gamma)} \gamma \lambda\right)
$$

is the unique solution of the following;

$$
\frac{d}{d m} \frac{d}{d x} u(x, \lambda)=\lambda u(x, \lambda)
$$




$$
\begin{gathered}
u^{\prime}(-\infty, \lambda)=0 \\
u(-\infty, \lambda)=1
\end{gathered}
$$

Note that $u(0-, \lambda)=\infty$.

EXAMPle 4. Let $m(x)=a\left(e^{a x}-1\right),(a>0)$ for $x \in \mathbf{R}$.

$$
u(x, \lambda)=\Phi_{1}\left(e^{a x} \lambda\right)
$$

is the unique solution of (3.11) with the boundary conditions (3.12) and (3.13). Note that $u(\infty, \lambda)=\infty$. Set $u_{1}(x, \lambda)=u(x, \lambda)$ and set

$$
u_{2}(x, \lambda)=u_{1}(x, \lambda) \int_{0}^{x} u_{1}(y, \lambda)^{-2} d y
$$

$u_{2}$ is a solution of (3.11) linearly independent of $u_{1}$. Since

$$
\begin{array}{ll}
u_{1}(0, \lambda)=\Phi_{1}(\lambda), & u_{1}^{\prime}(0, \lambda)=\Phi_{1}^{\prime}(\lambda) \\
u_{2}(0, \lambda)=0, & u_{2}^{\prime}(0, \lambda)=1 / \Phi_{1}(\lambda)
\end{array}
$$

solutions of (2.2) and (2.3) are given by

$$
\begin{aligned}
& \varphi(x, \lambda)=u_{1}(x, \lambda) / \Phi_{1}(\lambda)-a \lambda u_{2}(x, \lambda) \Phi_{1}^{\prime}(\lambda) \\
& \phi(x, \lambda)=u_{2}(x, \lambda) \Phi_{1}(\lambda)
\end{aligned}
$$

respectively. Setting

$$
\tilde{h}(\lambda):=\lim _{x \rightarrow-\infty} u_{2}(x, \lambda) / u_{1}(x, \lambda)=-\int_{-\infty}^{0} u_{1}(y, \lambda)^{-2} d y
$$

we have,

$$
\begin{aligned}
h(\lambda) & =-\lim _{x \rightarrow-\infty} \phi(x, \lambda) / \varphi(x, \lambda) \\
& =-\Phi_{1}(\lambda) /\left[\left\{\Phi_{1}(\lambda) \tilde{h}(\lambda)\right\}^{-1}-a \lambda \Phi_{1}^{\prime}(\lambda)\right] \\
& =\Phi_{1}(\lambda) / a \lambda \Phi_{1}^{\prime}(\lambda) .
\end{aligned}
$$

Here we used $\tilde{h}(\lambda)=-\infty$. 


\section{$\S 4$. Limit distributions of normalized hitting time}

In this section, we treat limit distribution of suitably normalized $\tau_{x}$ as $x \rightarrow l_{2}$ when the origin is a reflecting boundary. We can describe our result in a unified and simple form.

The following lemma can be easily proved by using (2.2) and (2.3).

LEMMA 2. Let $\varphi, \phi$ be the solution of (2.2) and (2.3), respectively, and let $\varphi^{(n)}(x, \lambda)=\frac{\partial^{n}}{\partial \lambda^{n}} \varphi(x, \lambda), \phi^{(n)}(x, \lambda)=\frac{\partial^{n}}{\partial \lambda^{n}} \phi(x, \lambda)$. These functions are absolutely continuous in $x$ and represented as:

$$
\begin{aligned}
\varphi^{(1)}(x, 0) & =\int_{0}^{x} m(y) d y \\
& \varphi^{(n)}(x, 0)=n \int_{0}^{x} \int_{0}^{y} \varphi^{(n-1)}(z, 0) m(d z) d y \\
= & n ! \int_{0}^{x} d y_{n} \int_{0}^{y_{n}}\left(m\left(y_{n}\right)-m\left(y_{n-1}\right)\right) d y_{n-1} \cdots \int_{0}^{y_{2}}\left(m\left(y_{2}\right)-m\left(y_{1}\right)\right) m\left(y_{1}\right) d y_{1}
\end{aligned}
$$

for $n>1$ and

$$
\begin{aligned}
& \phi^{(n)}(x, 0)=n \int_{0}^{x} \int_{0}^{y} \phi^{(n-1)}(z, 0) m(d z) d y \\
= & n ! \int_{0}^{x} d y_{n+1} \int_{0}^{y_{n+1}}\left(m\left(y_{n+1}\right)-m\left(y_{n}\right)\right) d y_{n} \cdots \int_{0}^{y_{2}}\left(m\left(y_{2}\right)-m\left(y_{1}\right)\right) d y_{1} .
\end{aligned}
$$

Note that if $l_{1}=-\infty$ and $m=0$ for $x<0$, then

$$
\begin{gathered}
E_{0}\left(\tau_{x}\right)=\varphi^{\prime}(x, 0) / \varphi^{2}(x, 0)=\varphi^{\prime}(x, 0)=\int_{0}^{x} m(y) d y \\
V_{0}\left(\tau_{x}\right)=\left\{\left(\varphi^{\prime}\right)^{2}-\varphi^{\prime \prime}\right\}(x, 0)=2 \int_{0}^{x} \int_{0}^{y} m(z)^{2} d z d y \\
E_{0}\left(\left(\tau_{x}-E_{0} \tau_{x}\right)^{3}\right)=\left\{\varphi^{(3)}-3 \varphi^{\prime \prime} \varphi^{\prime}+2\left(\varphi^{\prime}\right)^{3}\right\}(x, 0) \\
=12 \int_{0}^{x} \int_{0}^{y} m(z)\left(\int_{0}^{z} m(u)^{2} d u\right) d z d y .
\end{gathered}
$$

If $m \neq 0$ for $x<0$, then the representations of the moments by the speed measure are complicated. We will describe the representation in a special case in Lemmas 9 and 11 in Section 6. 
Denote $a_{k}(x)=\varphi^{(k)}(x, 0) / k$ ! and $b_{k}(x)=\phi^{(k)}(x, 0) / k$ ! for $k \geqq 0$. We have, by Lemma 2 ,

$$
\begin{aligned}
& a_{1}(x)=\int_{0}^{x} m(y) d y, \quad b_{1}(x)=\int_{0}^{x} d y \int_{0}^{y}(m(y)-m(z)) d z \\
& a_{k}(x)=\int_{0}^{x} \int_{0}^{y} a_{k-1}(z) m(d z) d y, \quad b_{k}(x)=\int_{0}^{x} \int_{0}^{y} b_{k-1}(z) m(d z) d y .
\end{aligned}
$$

Set $a(x)=a_{1}(x)$. Throughout this paper, we assume that

$$
a(x) \longrightarrow \infty \quad \text { as } \quad x \rightarrow l_{2} .
$$

We introduce the following assumption on $m \in \mathcal{M}$.

$\left(A_{\gamma}\right)(0 \leqq \gamma<\infty): \quad m(x) a(x) / \int_{0}^{x} m(y)^{2} d y \longrightarrow 1+\gamma$ as $x \rightarrow l_{2}$,

$$
\text { : } \quad m(x) a(x) / \int_{0}^{x} m(y)^{2} d y \longrightarrow \infty \quad \text { as } \quad x \rightarrow l_{2} .
$$

For any function $\Psi(\gamma)$ of $\gamma \in(0, \infty)$, we set $\Psi(0)=\lim _{\gamma \rightarrow 0} \Psi(\gamma)$ and $\Psi(\infty)=\lim _{\gamma \rightarrow \infty} \Psi(\gamma)$.

LEMMA 3. Under the assumption $\left(A_{\gamma}\right)$ for some $0 \leqq \gamma \leqq \infty$,

$$
\int_{0}^{x} a(y)^{n} m(y)^{2} d y / m(x) a(x)^{n+1} \longrightarrow \frac{1}{n+1+\gamma} \quad \text { as } x \rightarrow l_{2} \text {, for } n \geqq 0 \text {. }
$$

Proof. Integration by parts yields

$$
\begin{aligned}
& \int_{0}^{x} a(y)^{n} m(y)^{2} d y \\
= & a(x)^{n} \int_{0}^{x} m(y)^{2} d y-\int_{0}^{x} n a(y)^{n-1} m(y) \int_{0}^{y} m(z)^{2} d z d y .
\end{aligned}
$$

By the assumption $\left(A_{\gamma}\right)$ and L'hospital's rule, we have

$$
\begin{array}{r}
\int_{0}^{x} a(y)^{n-1} m(y) \int_{0}^{y} m(z)^{2} d z d y / \int_{0}^{x} a(y)^{n} m(y)^{2} d y \underset{1+\gamma}{\longrightarrow} \frac{1}{1+\gamma} \quad x \rightarrow l_{2} .
\end{array}
$$


By (4.5), (4.6) and the assumption, we have, for $\gamma<\infty$,

$$
\begin{aligned}
\frac{n+1+\gamma}{1+\gamma} \int_{0}^{x} a(y)^{n} m(y)^{2} d y & \sim a(x)^{n} \int_{0}^{x} m(y)^{2} d y \\
& \sim \frac{1}{1+\gamma} a(x)^{n+1} m(x) \quad \text { as } \quad x \rightarrow l_{2} .
\end{aligned}
$$

Also, we have, for $\gamma=\infty$,

$$
\int_{0}^{x} a(y)^{n} m(y)^{2} d y=o\left(a(x)^{n+1} m(x)\right) \quad \text { as } \quad x \rightarrow l_{2} .
$$

These give the conclusion of the lemma.

LEMMA 4. Under the assumption $\left(A_{\gamma}\right)$ for some $0 \leqq \gamma \leqq \infty$,

$$
a_{n+1}^{\prime}(x) / m(x) a(x)^{n} \longrightarrow \Gamma(\gamma) \gamma^{n+1} / n ! \Gamma(n+1+\gamma) \quad \text { as } \quad x \rightarrow l_{2}
$$

for any nonnegative integer $n$.

Proof. We prove by induction in $n$. For $n=0$, both sides of (4.7) are equal to 1 and the conclusion holds. Suppose that (4.7) holds for $n \leqq k-1$. By (4.4), we have

$$
a_{k+1}^{\prime}(x)=a_{k}(x) m(x)-\int_{0}^{x} a_{k}^{\prime}(y) m(y) d y .
$$

By (4.7) for $n=k-1$, L'hospital's rule gives

$$
a_{k}(x) / a(x)^{k} \longrightarrow \frac{\Gamma(\gamma) \gamma^{k}}{k ! \Gamma(k+\gamma)}
$$

and

$$
\int_{0}^{x} a_{k}^{\prime}(y) m(y) d y / \int_{0}^{x} a(y)^{k-1} m(y)^{2} d y \longrightarrow \frac{\Gamma(\gamma) \gamma^{k}}{(k-1) ! \Gamma(k+\gamma)} \quad \text { as } \quad x \rightarrow l_{2}
$$

Hence, by Lemma 3,

$$
\begin{aligned}
\int_{0}^{x} a_{k}^{\prime}(y) & m(y) d y / a(x)^{k} m(x) \\
& \longrightarrow \frac{\Gamma(\gamma) \gamma^{k}}{(k-1) ! \Gamma(k+\gamma)} \cdot \frac{1}{k+\gamma} \quad \text { as } \quad x \rightarrow l_{2}
\end{aligned}
$$

By $(4.8)-(4.10)$, we have

$$
a_{k+1}^{\prime}(x) / m(x) a(x)^{k} \longrightarrow \frac{\Gamma(\gamma) \gamma^{k+1}}{k ! \Gamma(k+1+\gamma)} \quad \text { as } \quad x \rightarrow l_{2} .
$$


Proposition 1. Under the assumption $\left(A_{\gamma}\right)$ for some $0 \leqq \gamma \leqq \infty$,

$$
\varphi(x, \lambda / a(x)) \longrightarrow \Phi_{\gamma}(\gamma \lambda) \quad \text { as } \quad x \rightarrow l_{2}
$$

uniformly in any compact set of $\lambda \geqq 0$.

Proof. Note that $\varphi(x, \lambda / a(x))=\sum_{n=0}^{\infty} \frac{a_{n}(x)}{a(x)^{n}} \lambda^{n}$. By Lemma 4,

$$
\frac{a_{n}(x)}{a(x)^{n}} \longrightarrow \frac{\Gamma(\gamma) \gamma^{n}}{n ! \Gamma(n+\gamma)} \quad \text { as } \quad x \rightarrow l_{2}
$$

We show that $\frac{a_{n}(x)}{a(x)^{n}} \leqq 1 / n$ ! for $n \geqq 1$ by induction in $n$. It is obvious for $n=1$. Assume that it holds for $n \leqq k$. By (4.4),

$$
\begin{aligned}
a_{k+1}(x) & =\int_{0}^{x} \int_{0}^{y} a_{k}(z) m(d z) d y \\
& =\int_{0}^{x} a_{k}(y) a^{\prime}(y) d y-\int_{0}^{x} \int_{0}^{y} a_{k}^{\prime}(z) a^{\prime}(z) d z d y \\
& \leqq \int_{0}^{x} a_{k}(y) a^{\prime}(y) d y \\
& \leqq \int_{0}^{x} a(y)^{k} a^{\prime}(y) d y / k ! \\
& =a(x)^{k+1} /(k+1) !
\end{aligned}
$$

Hence it holds for $n=k$. This yields

$$
\varphi(x, \lambda / a(x)) \longrightarrow \sum_{k=0}^{\infty} \frac{\Gamma(\gamma)(\gamma \lambda)^{n}}{k ! \Gamma(k+\gamma)}=\Phi_{\gamma}(\gamma \lambda) \quad \text { as } \quad x \rightarrow l_{2}
$$

uniformly in any compact set of $\lambda \geqq 0$.

THEOREM 1. Suppose that $l_{1}=-\infty$ and $m(x)=0$ for $x<0$, and assume $\left(A_{\gamma}\right)$ for some $0 \leqq \gamma \leqq \infty$. Then

$$
E_{\xi}\left(e^{-\lambda \tau_{x} / a(x)}\right) \rightarrow 1 / \Phi_{\gamma}(\gamma \lambda) \quad \text { as } \quad x \rightarrow l_{2}, \quad-\infty<\xi<l_{2} .
$$

Proof. If $l_{1}=-\infty, m(x)=0$, for $x<0$, then

$$
E_{\xi}\left(e^{-\lambda \tau_{x} / a(x)}\right)=\varphi(\xi, \lambda / a(x)) / \varphi(x, \lambda / a(x)), \quad-\infty<\xi \leqq x<l_{2} .
$$

Hence we have the conclusion by Proposition 1 . 
The above result is obtained for birth and death processes by Soloviev under a condition equivalent to

$$
a(x) m^{\prime}(x) / m(x)^{2} \longrightarrow \gamma \quad(0 \leqq \gamma \leqq \infty) \quad \text { as } \quad x \rightarrow l_{2},
$$

which is stronger than our assumption.

Remark 1. By L'hospital's rule,

$$
\left\{E_{\xi}\left(\tau_{x}\right)\right\}^{2} / V_{\xi}\left(\tau_{x}\right) \sim m(x)(a(x)-a(\xi)) / \int_{0}^{x} m(y)^{2} d y \longrightarrow 1+\gamma \quad \text { as } x \rightarrow l_{2}
$$

This is a probabilistic meaning of the quantity $\gamma$.

\section{$\S 5$. Convergence to self-similar process}

In this section, we investigate a problem on the convergence of hitting time processes. For this purpose, we prepare the following assumptions: $\left(C_{\gamma}\right)(0 \leqq \gamma<1): \quad l_{2}=\infty$ and $m(x) \sim x^{\gamma /(1-\gamma)} L(x) /(1-\gamma)$ as $x \rightarrow \infty$. $\left(C_{1}\right): \quad l_{2}=\infty$ and there is a function $s(x)$ regularly varying at $\infty$ and differentiable for large $x$ such that the derivative $s^{\prime}(x)$ is positive and monotone and

$$
m(s(x)) \sim e^{x} L\left(e^{x}\right) \quad \text { as } x \rightarrow \infty
$$

with some function $L$ slowly varying at $\infty$.

$\left(C_{\gamma}\right)(1<\gamma<\infty): \quad l_{2}<\infty$ and $m(x) \sim\left(l_{2}-x\right)^{\gamma /(1-\gamma)} K\left(l_{2}-x\right) /(\gamma-1)$ as $x \rightarrow l_{2}$ with some $K$ slowly varying at 0 .

$\left(C_{\infty}\right): \quad l_{2}<\infty$ and $m(x) \sim\left(l_{2}-x\right)^{-1} K\left(l_{2}-x\right)$ as $x \rightarrow l_{2}$ with some $K$ slowly varying at 0 .

Remark 2. If $0 \leqq \gamma<0$ and $\gamma \neq 1$, then $\left(C_{\gamma}\right)$ is equivalent to the following $\left(B_{\gamma}\right)$.

$\left(B_{\gamma}\right)(0 \leqq \gamma<1): l_{2}=\infty$ and $a(x) \sim(1-\gamma) x m(x)$ as $x \rightarrow \infty$, $\left(B_{\gamma}\right)(1<\gamma<\infty): \quad l_{2}<\infty$ and $a(x) \sim(\gamma-1)\left(l_{2}-x\right) m(x)$ as $x \rightarrow l_{2}$, which is obtained by Seneta [8; Theorem 2.1].

LEMMA 5. 1. If $\gamma=1$ or $\gamma=\infty$, then $\left(C_{\gamma}\right)$ implies the following $\left(B_{\gamma}\right)$. $\left(B_{1}\right): \quad l_{2}=\infty,\left(A_{1}\right)$ holds, and $a(x)=o(x m(x))$ as $x \rightarrow \infty$.

$\left(B_{\infty}\right): \quad l_{2}<\infty$ and $a(x)=o(m(x))$ and $\left(l_{2}-x\right) m(x)=o(a(x))$ as $x, \rightarrow l_{2}$.

2. If $0 \leqq \gamma \leqq \infty$ and $\gamma \neq 1$, then $\left(B_{\gamma}\right)$ implies $\left(A_{\gamma}\right)$. 
Proof. 1. Let $\gamma=1$ and let $r(x)=s^{-1}(x)$. Then

$$
m(x) \sim e^{r(x)} L\left(e^{r(x)}\right) \quad \text { as } x \rightarrow \infty .
$$

Since, for large $M$,

$$
\begin{aligned}
& a(x) \sim \int_{M}^{x} m(y) d y \\
= & \int_{e^{r(M)}}^{e^{r(x)}} \frac{m(s(\log u))}{r^{\prime}(s(\log u)) u} d u
\end{aligned}
$$

and

it holds that

$$
\int_{1}^{x} \frac{L(u)}{r^{\prime}(s(\log u))} d u \sim \frac{x L(x)}{r^{\prime}(s(\log x))} \quad \text { as } \quad x \rightarrow \infty
$$

$$
a(x) \sim e^{r(x)} L\left(e^{r(x)}\right) / r^{\prime}(x)
$$

By (5.1) and (5.2),

$$
a(x) / x m(x) \sim 1 / x r^{\prime}(x) \longrightarrow 0
$$

as $x \rightarrow \infty$. Since

$$
\begin{aligned}
\int_{s(\log M)}^{s(\log x)} m(y)^{2} d y & =\int_{M}^{x} m(s(\log u))^{2} s^{\prime}(\log u) u^{-1} d u \\
& \sim \int_{M}^{x} u L(u)^{2} s^{\prime}(\log u) d u \sim x^{2} L(x)^{2} s^{\prime}(\log x) / 2
\end{aligned}
$$

we have, by $(5.1)-(5.3)$, that

$$
a(s(\log x)) m(s(\log x)) / \int_{1}^{s(\log x)} m(y)^{2} d y \sim 2 .
$$

Hence $\left(B_{1}\right)$ holds. It is easy to show that $\left(C_{\infty}\right)$ implies $\left(B_{\infty}\right)$.

2. If $\left(B_{\gamma}\right)$ holds for some $0 \leqq \gamma<1$, then, by using L'hospital's rule, we have

$$
\begin{aligned}
& m(x) a(x) / \int_{0}^{x} m(y)^{2} d y \\
\sim & a(x)^{2} /(1-\gamma) x \int_{0}^{x} m(y)^{2} d y \\
\sim & a(x)\{2 x m(x)-a(x)\} /(1-\gamma) x^{2} m(x)^{2} \\
\sim & (1-\gamma) x m(x)\{(1+\gamma) x m(x)\} /(1-\gamma) x^{2} m(x)^{2} \\
= & 1+\gamma \quad \text { as } \quad x \rightarrow l_{2} .
\end{aligned}
$$


If $\left(B_{\gamma}\right)$ holds for some $1<\gamma<\infty$, then, we have

$$
\begin{aligned}
& m(x) a(x) / \int_{0}^{x} m(y)^{2} d y \\
\sim & a(x)^{2} /(\gamma-1)\left(l_{2}-x\right) \int_{0}^{x} m(y)^{2} d y \\
\sim & a(x)\left\{2\left(l_{2}-x\right) m(x)+a(x)\right\} /(\gamma-1)\left(l_{2}-x\right)^{2} m(x)^{2} \\
\sim & (\gamma-1)\left(l_{2}-x\right) m(x)\left\{(1+\gamma)\left(l_{2}-x\right) m(x)\right\} /(\gamma-1)\left(l_{2}-x\right)^{2} m(x)^{2} \\
= & 1+\gamma \quad \text { as } \quad x \rightarrow l_{2} .
\end{aligned}
$$

Here we used L'hospital's rule again. If $\left(B_{\infty}\right)$ holds, then, we have

$$
\begin{aligned}
& m(x) a(x) / \int_{0}^{x} m(y)^{2} d y \\
= & o\left\{a(x)^{2} /\left(l_{2}-x\right) \int_{0}^{x} m(y)^{2} d y\right\},
\end{aligned}
$$

and, using L'hospital's rule, we have

$$
\begin{aligned}
& a(x)^{2} /\left(l_{2}-x\right) \int_{0}^{x} m(y)^{2} d y \\
= & a(x)\left\{2\left(l_{2}-x\right) m(x)+a(x)\right\} /\left(l_{2}-x\right)^{2} m(x)^{2} \\
\sim & a(x)^{2} /\left(l_{2}-x\right)^{2} m(x)^{2} \\
\rightarrow & \infty \quad \text { as } \quad x \rightarrow l_{2} .
\end{aligned}
$$

We get the conclusion.

Remark 3. The assumption $\left(B_{1}\right)$ includes $\left(A_{1}\right)$. This is natural in the following sense. Let us assume

$$
a(x) \sim c x^{b} m(x) \quad \text { as } \quad x \rightarrow \infty \quad(c>0, \quad-\infty<b<1) .
$$

Then, we have by using L'hospital's rule

$$
\begin{aligned}
& m(x) a(x) / \int_{0}^{x} m(y)^{2} d y \\
\sim & a(x)^{2} / c x^{b} \int_{0}^{x} m(y)^{2} d y \\
\sim & {\left[2 c^{2} x^{2 b} m(x)^{2}-b c x^{b-1}\left\{c x^{b} m(x)\right\}^{2} / c^{2} x^{2 b} m(x)^{2}\right.} \\
\rightarrow & 2 \quad \text { as } \quad x \rightarrow l_{2} .
\end{aligned}
$$

Hence $\left(A_{1}\right)$ holds. 
We next note that the following limit theorem for the function $\phi$.

Proposition 2. 1. Under the assumption $\left(B_{\gamma}\right)$ with $0 \leqq \gamma<1$,

$$
\phi(x, \lambda / a(x)) / x \longrightarrow \sum_{n=0}^{\infty} \frac{\Gamma(2-\gamma) \gamma^{n}}{n ! \Gamma(n+2-\gamma)} \lambda^{n}=\Phi_{2-\gamma}(\gamma \lambda)
$$

as $x \rightarrow l_{2}$ uniformly in any compact set of $\lambda$.

2. Under the assumption $\left(B_{\gamma}\right)$ with $1 \leqq \gamma \leqq \infty$,

$$
\phi(x, \lambda / a(x)) / x \longrightarrow \sum_{n=0}^{\infty} \frac{\Gamma(\gamma) \gamma^{n}}{n ! \Gamma(n+\gamma)} \lambda^{n}=\Phi_{\gamma}(\gamma \lambda)
$$

as $x \rightarrow l_{2}$ uniformly in any compact set of $\lambda$.

Since $\left(B_{\gamma}\right)$ is equivalent to $\left(C_{\gamma}\right)$ for $\gamma \in[0, \infty) \backslash\{1\}, 1$ of Proposition 2 is immediately obtained by Kasahara's continuity theorem on Krein's correspondence ([4]). Let us observe the asymptotic behavior of $b_{n}(x)$ defined by (4.4) as $x \rightarrow \infty$.

Lemma 6. Assume $\left(B_{\gamma}\right)$ for some $1 \leqq \gamma \leqq \infty$. Then, for $k \geqq 1$,

$$
\int_{0}^{x} a(y)^{k-1} y m(y)^{2} d y / a(x)^{k} x m(x) \longrightarrow \frac{1}{k+\gamma} \quad \text { as } \quad x \rightarrow l_{2} .
$$

Proof. Integration by parts gives

$$
\begin{aligned}
& \int_{0}^{x} a(y)^{k-1} y m(y)^{2} d y \\
= & x a(x)^{k-1} \int_{0}^{x} m(y)^{2} d y \\
& -\int_{0}^{x}(k-1) a(y)^{k-2} y m(y) \int_{0}^{y} m(z)^{2} d z d y \\
& -\int_{0}^{x} a(y)^{k-1} \int_{0}^{y} m(z)^{2} d z d y .
\end{aligned}
$$

Since the assumption $\left(A_{\gamma}\right)$ holds, we have

$$
\begin{aligned}
x a(x)^{k-1} \int_{0}^{x} m(y)^{2} d y & \sim \frac{1}{1+\gamma} a(x)^{k} x m(x) & & \text { for } 1 \leqq \gamma<\infty \\
& =o\left(a(x)^{k} x m(x)\right) & & \text { for } \gamma=\infty
\end{aligned}
$$




$$
\int_{0}^{x} a(y)^{k-2} y m(y) \int_{0}^{y} m(z)^{2} d z d y / \int_{0}^{x} a(y)^{k-1} y m(y)^{2} d y \longrightarrow \frac{1}{1+\gamma}
$$

and

$$
\begin{aligned}
\int_{0}^{x} a(y)^{k-1} \int_{0}^{y} m(z)^{2} d z d y / & \int_{0}^{x} a(y)^{k} m(y) d y \\
& \longrightarrow \frac{1}{1+\gamma} \quad \text { as } \quad x \rightarrow l_{2} .
\end{aligned}
$$

By the assumption $\left(B_{\gamma}\right)$ with $1 \leqq \gamma<\infty$, we have

$$
\begin{aligned}
\int_{0}^{x} a(y)^{k} m(y) d y & =a(x)^{k+1} /(k+1) \\
& =o\left(a(x)^{k} x m(x)\right) \text { if } 1 \leqq \gamma<\infty .
\end{aligned}
$$

By the monotonicity of $a(x)$ and $m(x)$, we have

$$
\int_{0}^{x} a(y)^{k} m(y) d y \leqq a(x)^{k} x m(x) .
$$

By $(5.4)-(5.8)$

$$
\begin{aligned}
\frac{k+\gamma}{1+\gamma} \int_{0}^{x} a(y)^{k-1} y m(y)^{2} d y & \\
& \sim \frac{1}{1+\gamma} a(x)^{k} x m(x) \quad \text { as } \quad x \rightarrow l_{2} \text { for } \quad \gamma<\infty .
\end{aligned}
$$

By (5.4) - (5.7) and (5.9)

$$
\int_{0}^{x} a(y)^{k-1} y m(y)^{2} d y=o\left(a(x)^{k} x m(x)\right) \quad \text { as } x \rightarrow l_{2} \quad \text { for } \quad \gamma=\infty .
$$

Hence,

$$
\int_{0}^{x} a(y)^{k-1} y m(y)^{2} d y / a(x)^{k} x m(x) \longrightarrow \frac{1}{k+\gamma} \quad \text { as } \quad x \rightarrow l_{2} .
$$

LEMma 7. Under the assumption $\left(B_{\gamma}\right)$ for some $1 \leqq \gamma \leqq \infty$, it holds for any $n \geqq 1$ that

$$
b_{n}(x) / x a(x)^{n} \sim b_{n}^{\prime}(x) / n a(x)^{n-1} x m(x) \rightarrow \frac{\Gamma(\gamma) \gamma^{n}}{n ! \Gamma(n+\gamma)}
$$

as $x \rightarrow l_{2}$. 
Proof. We prove by induction in $n$. For $n=1,(5.10)$ holds by

$$
\begin{aligned}
b_{1}^{\prime}(x) / x m(x) & =\{x m(x)-a(x)\} / x m(x) \rightarrow 1, \\
b_{1}(x) / x a(x) & \sim\{x m(x)-a(x)\} /\{x m(x)+a(x)\} \rightarrow 1 \quad \text { as } x \rightarrow l_{2} .
\end{aligned}
$$

Suppose that (5.10) holds for $n \leqq k$. By L'hospital's rule, we have

$$
\begin{aligned}
& b_{k+1}(x) / x a(x)^{k+1} \\
\sim & b_{k+1}^{\prime}(x) /\left\{a(x)^{k+1}+(k+1) a(x)^{k} x m(x)\right\} \\
\sim & \left\{b_{k}(x) m(x)-\int_{0}^{x} b_{k}^{\prime}(y) m(y) d y\right\} /\left\{a(x)^{k+1}+(k+1) a(x)^{k} x m(x)\right\} \\
\sim & \frac{\Gamma(\gamma) \gamma^{k}}{(k+1) ! \Gamma(k+\gamma)}-\frac{\Gamma(\gamma) \gamma^{k}}{(k+1)(k-1) ! \Gamma(k+\gamma)(k+\gamma)} \\
= & \frac{\Gamma(\gamma) \gamma^{k+1}}{(k+1) ! \Gamma(k+1+\gamma)} .
\end{aligned}
$$

Here, we used the induction hypothesis, $\left(B_{\gamma}\right),(4.4)$, and Lemma 6 .

Proof of 2 of Proposition 2. Since $b_{1}(x) / x a_{1}(x) \leqq 1$, as in the proof of Proposition 1, we can show that

$$
b_{n}(x) / x a(x)^{n} \leqq 1 / n !
$$

Hence by Lemmas 6 and 7, we get the conclusion.

Remark 4. Any $\varphi$ and $\phi$ satisfy

$$
1 \leqq \varphi(x, \lambda), \quad \phi(x, \lambda) / x \leqq e^{a(x) \lambda} \text { for } x, \lambda \geqq 0,
$$

which is easily seen by the proofs of Propositions 1 and 2 .

THEOREM 2. Assume that $l_{1}=-\infty$, that $\left(C_{\gamma}\right)$ holds for some $0 \leqq \gamma<$ 1 and that

$$
|m(-x)| / m(x) \rightarrow c \in[0, \infty) \quad \text { as } x \rightarrow \infty
$$

Then,

$$
\begin{aligned}
& \lim _{x \rightarrow \infty} E_{\xi}\left[e^{-\lambda \tau_{t x} / a(x)}\right] \\
= & 1 /\left\{\Phi_{\gamma}\left(\gamma \lambda t^{(1-\gamma)^{-1}}\right)+(c \lambda)^{1-\gamma} t \Phi_{2-\gamma}\left(\gamma \lambda t^{(1-\gamma)^{-1}}\right) / E_{\gamma}\right\}
\end{aligned}
$$


for $t, \lambda \geqq 0$ and $\xi \in \mathbf{R}$. Namely, the normalized generalized hitting time process $\left\{\tau_{t x} / a(x)\right\}$ with any starting point converges in finite dimensional distributions to a self-similar process $\left\{\tau_{t}^{0}\right\}$ with exponent $(1-\gamma)^{-1}$ with independent increments as $x \rightarrow \infty$, and $\left\{\tau_{t}^{0}\right\}$ is the hitting time process starting at the origin of the generalized diffusion process with speed measure

$$
m^{0}(x)= \begin{cases}x^{\gamma /(1-\gamma)} /(1-\gamma) & x \geqq 0 \\ -c|x|^{\gamma /(1-\gamma)} /(1-\gamma) & x<0\end{cases}
$$

Proof. By the assumption $\left(C_{\gamma}\right)$ with $0 \leqq \gamma<1$ and Propositions 1 and 2, we have

$$
\varphi(x, \lambda / a(x)) \rightarrow \Phi_{\gamma}(\gamma \lambda), \quad \phi(x, \lambda / a(x)) / x \rightarrow \Phi_{2-\gamma}(\gamma \lambda),
$$

as $x \rightarrow \infty$. These convergences are uniform in any compact set of $\lambda \geqq 0$. Since $a(t x) / a(x) \rightarrow t^{(1-\gamma)^{-1}}$ as $x \rightarrow \infty$, for any $t \geqq 0$,

$$
\begin{aligned}
& \varphi(t x, \lambda / a(x))=\varphi(t x, \lambda a(t x) / a(t x) a(x)) \rightarrow \Phi_{\gamma}\left(\gamma \lambda t^{(1-\gamma)^{-1}}\right), \\
& t \phi(t x, \lambda a(t x) / a(t x) a(x)) / t x \rightarrow t \Phi_{2-\gamma}\left(\gamma \lambda t^{(1-\gamma)^{-1}}\right) \quad \text { as } \quad x \rightarrow l_{2} .
\end{aligned}
$$

Since

$$
x m(t x) / a(x) \rightarrow-c|t|^{\gamma /(1-\gamma)} /(1-\gamma) \quad \text { as } x \rightarrow \infty \text { for } t<0
$$

we have, by Kasahara's continuity theorem,

$$
h(\lambda / a(x)) / x \rightarrow E_{\gamma}(c \lambda)^{\gamma-1} \quad \text { as } \quad x \rightarrow \infty .
$$

Hence we get, for $t>0$ and $\xi \in \mathbf{R}$ that

$$
\begin{aligned}
& E_{\xi}\left(e^{-\lambda \tau_{t x} / a(x)}\right) \\
= & u(\xi, \lambda / a(x)) / u(x t, \lambda / a(x)) \\
\rightarrow & 1 /\left\{\Phi_{\gamma}\left(\gamma \lambda t^{(1-\gamma)^{-1}}\right)+(c \lambda)^{1-\gamma} t \Phi_{2-\gamma}\left(\gamma \lambda t^{(1-\gamma)^{-1}}\right) / E_{\gamma}\right\} \\
= & 1 / u^{0}(t, \lambda)
\end{aligned}
$$

as $x \rightarrow \infty$, where $1 / u^{0}(t, \lambda)$ is the Laplace transform of $\tau_{t}^{0}$ by Example 2 in Section 3. Since $\left\{\tau_{x}\right\}$ has independent increments, $\left\{\tilde{\tau}_{t}^{x}\right\}=\left\{\tau_{t x} / a(x)\right\}$ also has independent increments. By (2.5), the finite dimensional distributions of $\left\{\tilde{\tau}_{t}^{x}\right\}$ are determined by $\tilde{u}_{x}(t, \lambda)=u(x t, \lambda / a(x))$. Hence, we get the convergence of finite dimensional distributions. 
Remark 5. (i) In the case $\gamma=0$ and $c=0$, the limit distribution is exponential by (3.2).

(ii) In the case $\gamma=\frac{1}{2}$ and $c=1$ in Theorem 2, the limit process is a one-sided stable process with exponent $\frac{1}{2}$. The Laplace transform is given by $e^{-t(\lambda / 2)^{1 / 2}}$. It may be an interesting problem for what measure $m$, there appear other stable distributions.

Remark 6. Self-similar processes with independent increments are extensively studied by Sato [7].

The following theorem treats the case $c=\infty$ in Theorem 2 .

ThEOREM 3. Let $\tilde{m}(x)=-m((-x)+)+m(0)-m(0-)$ and $\tilde{a}(x)=$ $\int_{0}^{x}|m(-y)| d y$. Assume that $\tilde{m}$ satisfies $\left(C_{\gamma}\right)$ for some $0 \leqq \gamma<1$ and that

$$
a(x) / \tilde{a}(x) \rightarrow 0 \quad \text { as } x \rightarrow \infty
$$

Then,

$$
\lim _{x \rightarrow \infty} E_{\xi}\left[e^{-\lambda \tau_{t x} / \tilde{a}(x)}\right]=1 /\left(1+t \lambda^{1-\gamma} / E_{\gamma}\right)
$$

for $t, \lambda \geqq 0$ and $\xi \in \mathbf{R}$, that is, the normalized generalized hitting time process $\left\{\tau_{t x} / \tilde{a}(x)\right\}$ with any starting point converges, as $x \rightarrow \infty$, in finite dimensional distributions to a self-similar process $\left\{\tau_{t}^{0}\right\}$ with exponent $(1-$ $\gamma)^{-1}$ with independent increments starting at the origin, $\left\{\tau_{t}^{0}\right\}$ is the hitting time process starting at the origin of the generalized diffusion process with speed measure

$$
m^{0}(x)= \begin{cases}0 & x \geqq 0 \\ -|x|^{\gamma /(1-\gamma)} /(1-\gamma) & x<0\end{cases}
$$

Proof. By (5.11), Remark 4 and the assumption $\left(C_{\gamma}\right)$ with $0 \leqq \gamma<1$, we have

$$
\begin{aligned}
& \varphi(x t, \lambda / \tilde{a}(x)) \\
= & \varphi(x t,(\lambda / \tilde{a}(x t))(\tilde{a}(x t) / \tilde{a}(x))) \longrightarrow 1, \\
& x^{-1} \phi(x t, \lambda / \tilde{a}(x)) \\
= & (t x)^{-1} \phi(x t,(\lambda / \tilde{a}(x t))(\tilde{a}(x t) / \tilde{a}(x))) \longrightarrow 1 .
\end{aligned}
$$


We have, by Kasahara's continuity theorem,

$$
x^{-1} h(\lambda / \tilde{a}(x)) \longrightarrow E_{\gamma} \lambda^{\gamma-1} .
$$

Hence,

$$
\begin{aligned}
E_{\xi}\left(e^{-\lambda \tau_{t x} / \tilde{a}(x)}\right) & =u(\xi, \lambda / \tilde{a}(x)) / u(t x, \lambda / \tilde{a}(x)) \\
& \rightarrow 1 /\left(1+t \lambda^{1-\gamma} / E_{\gamma}\right) \quad \text { as } \quad x \rightarrow \infty
\end{aligned}
$$

Since $\left\{\tau_{t x} / \tilde{a}(x)\right\}$ has independent increments, the convergence of finite dimensional distributions is obvious.

LEMMA 8. Let $0 \leqq p \leqq \infty$ and

$$
m_{p}(t)=\left\{\begin{array}{lll}
0 & \text { for } & 0 \leqq t<p \\
\infty & \text { for } & p \leqq t<\infty
\end{array}\right.
$$

Assume that $l_{1}=-\infty$ and

$$
x|m(-t x)| / a(x) \longrightarrow m_{p}(t) \quad \text { as } \quad x \rightarrow \infty .
$$

Then,

$$
h(\lambda / a(x)) / x \rightarrow p \quad \text { as } \quad x \rightarrow \infty .
$$

Proof. By Example 1 in Section 3, $h(\lambda / a(x)) / x$ corresponds to $x m(-t x) / a(x)$ and $h(\lambda)=p$ corresponds to $-m_{p}(-t)$. Hence by Kasahara's continuity theorem, the conclusion is obvious.

THEOREM 4. Assume $\left(C_{1}\right)$ and that

$$
x|m(-t x)| / a(x) \longrightarrow m_{p}(t)
$$

as $x \rightarrow \infty$ for some $p \in[0, \infty]$. Then

$$
E_{\xi}\left(e^{-\lambda \tau_{s(x+t)} / a(s(x))}\right) \longrightarrow 1 /(1+1 / p) \Phi_{1}\left(e^{t} \lambda\right) \quad \text { as } x \rightarrow \infty
$$

for $t, \xi \in \mathbf{R}$ and $\lambda \geqq 0$. Especially, in case $p=\infty$, the normalized generalized hitting time process $\left\{\tau_{s(x+t)} / a(s(x))\right\}$ with any starting point converges in finite dimensional distributions to a process $\left\{\tau_{t}^{0}\right\}$ with independent increments, which is the hitting time process of the generalized diffusion process with speed measure $m^{0}(t)=e^{t}, t \in \mathbf{R}$ and starting point $-\infty$. 
Proof. By the assumption $\left(C_{1}\right)$ and Lemma 5 , the assumption $\left(B_{1}\right)$ is satisfied. By Propositions 1, 2 and Lemma 8, we get

$$
\begin{aligned}
& \varphi(x, \lambda / a(x)), \quad \phi(x, \lambda / a(x)) / x \longrightarrow \Phi_{1}(\lambda), \\
& h(\lambda / a(x)) / x \longrightarrow p
\end{aligned}
$$

as $x \rightarrow \infty$. These convergences are uniform in any compact set of $\lambda \geqq 0$. Since $a(s(x+t)) / a(s(x)) \rightarrow e^{t}$ as $x \rightarrow \infty$, for any $t \in \mathbf{R}$,

$$
\begin{aligned}
& \varphi(s(x+t), \lambda / a(s(x))) \\
= & \varphi(s(x+t),\{\lambda / a(s(x+t))\}\{a(s(x+t)) / a(s(x))\}) \\
\rightarrow & \Phi_{1}\left(e^{t} \lambda\right), \\
& \phi(s(x+t), \lambda / a(s(x))) / s(x+t) \\
= & \phi(s(x+t),\{\lambda / a(s(x+t))\}\{a(s(x+t)) / a(s(x))\}) / s(x+t) \\
\rightarrow & \Phi_{1}\left(e^{t} \lambda\right),
\end{aligned}
$$

and

$$
h(\lambda / a(s(x+t))) / s(x+t) \longrightarrow p, \quad \text { as } \quad x \rightarrow \infty .
$$

Hence, for any $t \in \mathbf{R}$, we get (5.12). As in the proof of Theorem 2, the convergence of the finite dimensional distributions is obvious in case $p=\infty$. By Example 4 in Section 3, the limit process is the hitting time process of a generalized diffusion with speed measure $m^{0}(t)=e^{t}$ and starting point $-\infty$.

The following theorem treats the case $p=0$ in Theorem 4 .

THEOREM 5. Let $\tilde{m}(x)=-m((-x)+)+m(0)-m(0-)$ and $\tilde{a}(x)=$ $\int_{0}^{x}|m(-y)| d y$. Assume that $\tilde{m}$ satisfies $\left(C_{1}\right)$ and that $l_{2}=\infty$ and, for any $t \geqq 0$,

$$
a(t x) / \tilde{a}(x) \longrightarrow 0 \quad \text { as } \quad x \rightarrow \infty .
$$

Then

$$
\lim _{x \rightarrow \infty} E_{\xi}\left[e^{-\lambda \tau_{t x} / \tilde{a}(x)}\right]=1 /(1+t) \quad \text { for } t, \lambda \geqq 0 \text { and } \xi \in \mathbf{R} .
$$

Remark 7. The limit distribution coincides with the limit distribution in Theorem 3 with $\gamma=1$. 
Proof of Theorem 5. By the assumption $\left(C_{1}\right)$, we get

$$
x|m(-t x)| / \tilde{a}(x) \longrightarrow m_{1}(t) \quad \text { as } \quad x \rightarrow \infty .
$$

Hence, by Lemma 8, we have

$$
h(\lambda / \tilde{a}(x)) / x \longrightarrow 1
$$

as $x \rightarrow \infty$. We have, by (5.13) and Remark 4 , that

$$
\varphi(t x, \lambda / \tilde{a}(x)), \phi(t x, \lambda / \tilde{a}(x)) / t x \longrightarrow 1 .
$$

Hence we have (5.14).

Remark 8. Let

$$
m(x) \sim e^{r(x)} L\left(e^{r(x)}\right)
$$

and

$$
m(-x) \sim e^{r_{1}(x)} L_{1}\left(e^{r_{1}(x)}\right) \quad \text { as } \quad x \rightarrow \infty,
$$

where, $r$ and $r_{1}$ are regularly varying at $\infty$, differentiable for large $x>0$, the derivatives $r^{\prime},\left(r_{1}\right)^{\prime}$ are positive and monotone, and $L$ and $L_{1}$ are slowly varying at $\infty$. Then, by (5.2),

$$
a(x) \sim e^{r(x)} L\left(e^{r(x)}\right) / r^{\prime}(x) .
$$

Hence

$$
x|m(-t x)| / a(x) \sim x e^{r_{1}(t x)} L_{1}\left(e^{r_{1}(t x)}\right) r^{\prime}(x) / e^{r(x)} L\left(e^{r(x)}\right) .
$$

Now, set

$$
r(x)=x^{\alpha} K(x), \quad r_{1}(x)=x^{\beta} K_{1}(x)
$$

where $K$ and $K_{1}$ are slowly varying at $\infty$. Let $t>0$.

(i) If $\alpha<\beta$, then $a(t x) / \tilde{a}(x) \rightarrow 0$ and $x|m(-t x)| / a(x) \rightarrow \infty$ as $x \rightarrow \infty$.

(ii) If $\alpha>\beta$, then $\tilde{a}(t x) / a(x), x|m(-t x)| / a(x) \rightarrow 0$ as $x \rightarrow \infty$.

(iii) If $\alpha=\beta$ and $\lim _{x \rightarrow \infty} K(x) / K_{1}(x) \rightarrow \kappa \in(0, \infty]$, then, for large $x$,

$$
t^{\alpha} K_{1}(t x)-K(x)\left\{\begin{array}{lll}
<0 & \text { if } & t<\kappa^{1 / \alpha} \\
>0 & \text { if } & t>\kappa^{1 / \alpha}
\end{array}\right.
$$

and hence

$$
x|m(-t x)| / a(x) \rightarrow m_{\kappa^{1 / \alpha}}(t) .
$$


(iv) If $\alpha=\beta$ and $\lim _{x \rightarrow \infty} K(x) / K_{1}(x) \rightarrow 0$, then

$$
a(t x) / \tilde{a}(x) \longrightarrow 0 .
$$

THEOREM 6. Assume $\left(C_{\gamma}\right)$ with $1<\gamma \leqq \infty$. Then, for any $t<0$ and $\xi \in \mathbf{R}$,

$$
\begin{aligned}
& E_{\xi}\left(e^{-l \tau_{l_{2}-y|t|} / a\left(l_{2}-y\right)}\right) \\
\rightarrow & \left\{\begin{array}{lll}
1 /\left(1+l_{2} /\left|l_{1}\right|\right) \Phi_{\gamma}\left(\gamma \lambda|t|^{1 /(1-\gamma)}\right) & \text { for } & 1<\gamma<\infty \\
1 /\left(1+l_{2} /\left|l_{1}\right|\right) e^{\lambda} & \text { for } & \gamma=\infty
\end{array}\right.
\end{aligned}
$$

as $y \downarrow 0$. Especially, in case $l_{1}=-\infty$ and $1<\gamma<\infty$, the normalized generalized hitting time process $\left\{\tau_{l_{2}-y|t|} / a\left(l_{2}-y\right)\right\}$ converges in finite dimensional distributions, as $y \downarrow 0$, to the hitting time process of a generalized diffusion with speed measure

$$
m^{0}(x)= \begin{cases}|x|^{\gamma /(1-\gamma)} /(\gamma-1), & x<0 \\ \infty, & x>0\end{cases}
$$

and starting point $-\infty$.

Proof. Note that

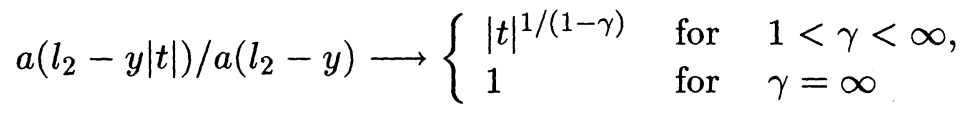

as $y \downarrow 0$. By Proposition 2, we have

$$
\begin{aligned}
& \phi\left(l_{2}-y|t|, \lambda / a\left(l_{2}-y\right)\right) \\
= & \phi\left(l_{2}-y|t|,\left\{\lambda / a\left(l_{2}-y|t|\right\}\left\{a\left(l_{2}-y|t|\right) / a\left(l_{2}-y\right)\right\}\right)\right. \\
\rightarrow & \left\{\begin{array}{lll}
l_{2} \Phi_{\gamma}\left(\gamma \lambda|t|^{1 /(1-\gamma)}\right) & \text { for } & 1<\gamma<\infty, \\
l_{2} e^{\lambda} & \text { for } & \gamma=\infty
\end{array}\right.
\end{aligned}
$$

as $x \rightarrow l_{2}$. By Proposition 1, we get

$$
\begin{aligned}
& \varphi\left(l_{2}-y|t|, \lambda / a\left(l_{2}-y\right)\right) \\
= & \varphi\left(l_{2}-y|t|,\left\{\lambda / a\left(l_{2}-y|t|\right\}\left\{a\left(l_{2}-y|t|\right) / a\left(l_{2}-y\right)\right\}\right)\right. \\
\rightarrow & \left\{\begin{array}{lll}
\Phi_{\gamma}\left(\gamma \lambda|t|^{1 /(1-\gamma)}\right) & \text { for } & 1<\gamma<\infty, \\
e^{\lambda} & \text { for } & \gamma=\infty
\end{array}\right.
\end{aligned}
$$


as $x \rightarrow l_{2}$. We have $h(\lambda / a(x)) \rightarrow\left|l_{1}\right|$. Hence, we get (5.15). If $1<\gamma<\infty$, then by Example 3 in Section 3, $1 / \Phi_{\gamma}\left(\gamma \lambda|t|^{1 /(1-\gamma)}\right)$ is the Laplace transform of the hitting time process of the generalized diffusion with speed measure

$$
m^{0}(x)= \begin{cases}(\gamma-1)|x|^{\gamma /(1-\gamma)} & x<0 \\ 0 & x>0\end{cases}
$$

and starting point $-\infty$. If $l_{1}=-\infty$, then the limit distribution has total mass 1 and hence we have also the convergence of finite dimensional distributions as the proof of Theorem 2 shows. The proof is complete.

We can prove Theorem 6 except the case $\gamma=\infty$ using the following Theorem 7, a version of Kasahara's continuity theorem. We can also prove Theorems 2 and 3 using Kasahara's continuity theorem. These proofs allow us to recognize the meaning of the convergence more clearly. However, in this paper, we make use of Propositions 1 and 2 in our proofs for unification of the method.

THEOREM 7. Let $m$ be a nonnegative and non-decreasing right continuous function on $\left(-\infty, l_{2}\right)$ satisfying $\left|\int_{-\infty}^{x} m(y) d y\right|<\infty$ for $x \in\left(-\infty, l_{2}\right)$ and $\varphi$ be the unique solution of the following equation:

$$
\varphi(x, \lambda)=1+\lambda \int_{(-\infty, x)}(x-y) \varphi(y, \lambda) d m(y) .
$$

For $n=1,2, \ldots$, let $m_{n}(x)$ be a non-decreasing right continuous function on $\left(l_{n 1}, l_{n 2}\right)$ such that there is $c_{n} \in\left(l_{n 1}, l_{n 2}\right)$ satisfying $m_{n}\left(c_{n}-\right)=0$. Let $a_{n}, b_{n} \in \mathbf{R}$ and let $\varphi_{n}(x, \lambda)$ be the solution of the following equation:

$$
\begin{aligned}
\varphi_{n}(x, \lambda) & =a_{n}+b_{n} x+\lambda \int_{\left[c_{n}, x\right)}(x-y) \varphi_{n}(y, \lambda) d m_{n}(y) \quad \text { for } c_{n} \leqq x<l_{n 2} \\
& =a_{n}+b_{n} x-\lambda \int_{\left[x, c_{n}\right)}(x-y) \varphi_{n}(y, \lambda) d m_{n}(y) \quad \text { for } l_{n 1}<x<c_{n}
\end{aligned}
$$

Suppose that

$$
\lim _{n \rightarrow \infty} \sup _{x}\left|\int_{\left[c_{n}, x\right)} m_{n}(y) d y\right|<\infty,
$$

$a_{n} \rightarrow a, c_{n} \rightarrow-\infty$ as $n \rightarrow \infty, \sup _{n}\left|b_{n} c_{n}\right|<\infty$, and

$$
\lim _{n \rightarrow \infty} m_{n}(x)=m(x)
$$


for continuity points $x \in\left(-\infty, l_{2}\right)$ of $m$. Then,

$$
\lim _{n \rightarrow \infty} \varphi_{n}(x, \lambda)=a \varphi(x, \lambda)
$$

uniformly in any compact set of $(x, \lambda)$ with $x<l_{2}$ and $\lambda \geqq 0$.

We can prove this theorem similarly to the proof of Kasahara's theorem.

\section{$\S 6$. Convergence to $N(0,1)$}

Theorem 6 and (3.3) show that the limit distribution is degenerate to one point in case $\gamma=\infty$. In this case, we get convergence to normal distribution by changing the normalization.

THEOREM 8. Assume that $l_{1}=-\infty$ and $m(x)=0$ for $x<0$. Then the distribution of $\tilde{\tau}_{x}=\left(\tau_{x}-E_{0} \tau_{x}\right) /\left(V_{0} \tau_{x}\right)^{1 / 2}$ converges to $N(0,1)$ as $x \rightarrow l_{2}$ if and only if

$$
\begin{aligned}
& \left\{\int_{o}^{x} \int_{0}^{y} m(z)\left(\int_{0}^{z} m(u)^{2} d u\right) d z d y\right\}^{2} \\
& =o\left(\left\{\int_{0}^{x} \int_{0}^{y} m(z)^{2} d z\right\}^{3}\right) \quad \text { as } x \rightarrow l_{2}
\end{aligned}
$$

Proof. If $l_{1}=-\infty$ and $m(x)=0$ for $x<0$, then (6.1) is equivalent to the condition $E_{0}\left(\tilde{\tau}_{x}\right)^{3} \rightarrow 0$ as $x \rightarrow l_{2}$. The conclusion is obvious by the result of [5] for $C E_{+}$distributions (convolutions of exponential distributions on $R_{+}$) that the distribution of $\tilde{\tau}_{x} \rightarrow N(0,1)$ as $x \rightarrow l_{2}$ if and only if $E_{0}\left(\tilde{\tau}_{x}\right)^{3} \rightarrow 0$ as $x \rightarrow l_{2}$. Refer to [10] for definition of $C E_{+}$distribution.

Remark 9. It is shown in [5] that if $l_{1}=-\infty$ and $m(x)=0$ for $x<0$, then (6.1) implies $V_{0}\left(\tau_{x}\right) /\left(E_{0}\left(\tau_{x}\right)\right)^{2} \rightarrow 0$ as $x \rightarrow l_{2}$, that is, $\gamma=\infty$. It is an interesting problem what kind of limit distributions appear when $\gamma=\infty$ and (6.1) is not satisfied.

Set $M(x)=\int_{0}^{x} m(y)^{2} d y$.

Corollary 1. Suppose that $l_{1}=-\infty, m(x)=0$ for $x<0$,

$$
\int_{0}^{x} M(y) d y \longrightarrow \infty \quad \text { as } \quad x \rightarrow l_{2}
$$


and

$$
\left(\int_{0}^{x} m(y) M(y) d y\right)^{2}=o\left(M(x)^{2} \int_{0}^{x} M(y) d y\right) \quad \text { as } x \rightarrow l_{2} .
$$

Then the distribution of $\tilde{\tau}_{x}$ converges to $N(0,1)$ as $x \rightarrow l_{2}$.

Proof. (6.1) is satisfied in case that $\int_{0}^{x} \int_{0}^{y} m(z) M(z) d z d y$ is bounded. So, we assume that $\int_{0}^{x} \int_{0}^{y} m(z) M(z) d z d y$ tends to $\infty$ as $x \rightarrow l_{2}$. By L'hospital's rule, (6.2) and (6.3), we have

$$
\begin{aligned}
& \int_{0}^{x} \int_{0}^{y} m(z) M(z) d z d y /\left\{\int_{0}^{x} M(y) d y\right\}^{3 / 2} \\
\sim & \int_{0}^{x} m(z) M(z) d z /\left\{\frac{3}{2} M(x)\left(\int_{0}^{x} M(y) d y\right)^{1 / 2}\right\} \longrightarrow 0
\end{aligned}
$$

as $x \rightarrow l_{2}$.

Corollary 2. Suppose (6.2) and

$$
\left.M(x)=o\left(m(x)\left\{\int_{0}^{x} M(y) d y\right)\right\}^{1 / 2}\right) \quad \text { as } \quad x \rightarrow l_{2} .
$$

Then (6.3) holds.

Proof. By L'hospital's rule, we have

$$
\begin{aligned}
& \int_{0}^{x} m(z) M(z) d z /\left\{M(x)\left(\int_{0}^{x} M(y) d y\right)^{1 / 2}\right\} \\
\sim & m(x) M(x) /\left\{m(x)^{2}\left(\int_{0}^{x} M(y) d y\right)^{1 / 2}+\frac{1}{2} M(x)^{2}\left(\int_{0}^{x} M(y) d y\right)^{-1 / 2}\right\} \\
= & o(1)
\end{aligned}
$$

as $x \rightarrow l_{2}$. Hence (6.3) holds.

Remark 10. Assume $\left(C_{\infty}\right)$, that is $m(x) \sim\left(l_{2}-x\right)^{-1} L\left(l_{2}-x\right)$ as $x \rightarrow l_{2}$, where $l_{2}<\infty$ and $L$ is slowly varying at 0 . Then we have

$$
M(x) \sim\left(l_{2}-y\right)^{-1} L\left(l_{2}-y\right)^{2}
$$


and

$$
\{M(x) / m(x)\}^{2} \sim L\left(l_{2}-x\right)^{2}=o\left(\int_{0}^{x}\left(l_{2}-y\right)^{-1} L\left(l_{2}-y\right)^{2} d y\right) \quad \text { as } x \rightarrow l_{2} .
$$

Hence (6.2) and (6.4) hold in this case.

We use the notations $f^{\prime}, f^{\prime \prime}, f^{(n)}$ for the 1st, 2nd and $n$th derivatives, respectively, in the variable $\lambda$ of a function $f$ of $(x, \lambda)$.

LEMMA 9.

$$
x \varphi^{\prime}(x, 0)-\phi^{\prime}(x, 0)=2 \int_{0}^{x} \int_{0}^{y} m(z) d z d y
$$

and

$$
\begin{aligned}
& x \varphi^{\prime \prime}(x, 0)-\phi^{\prime \prime}(x, 0) \\
= & 4 \int_{0}^{x} m(y) \int_{0}^{y} \int_{0}^{z} m(u) d u d z d y-4 \int_{0}^{x} \int_{0}^{y} \int_{0}^{x} m(u)^{2} d u d z d y .
\end{aligned}
$$

Proof. By Lemma 2, we have

$$
\begin{aligned}
x \varphi^{\prime}(x, 0)-\phi^{\prime}(x, 0) & =\int_{0}^{x}(x-y) m(y) d y+\int_{0}^{x} \int_{0}^{y} m(z) d z d y \\
& =2 \int_{0}^{x} \int_{0}^{y} m(z) d z d y .
\end{aligned}
$$

Also, we have, by Lemma 2, that

$$
\varphi^{\prime \prime}(x, 0)=\varphi^{\prime}(x, 0)^{2}-2 \int_{0}^{x} \int_{0}^{y} m(z)^{2} d z d y
$$

and

$$
\phi^{\prime \prime}(x, 0)=2 \int_{0}^{x} d y \int_{0}^{y}(m(y)-m(z)) d z \int_{0}^{z}(m(z)-m(u)) d u .
$$

By integration by parts, we have

$$
\begin{aligned}
& \phi^{\prime \prime}(x, 0) \\
= & 2 \int_{0}^{x} m(y) \int_{0}^{y} z m(z) d z d y-2 \int_{0}^{x} \int_{0}^{y} z m(z)^{2} d z d y
\end{aligned}
$$




$$
\begin{aligned}
& -2 \int_{0}^{x} m(y) \int_{0}^{y} \int_{0}^{z} m(u) d u d z d y+2 \int_{0}^{x} \int_{0}^{y} m(z) \int_{0}^{z} m(u) d u d z d y \\
= & 2 x \int_{0}^{x} m(y) \int_{0}^{y} m(z) d z d y-2 x \int_{0}^{x} \int_{0}^{y} m(z)^{2} d z \\
& +4 \int_{0}^{x} \int_{0}^{y} \int_{0}^{z} m(u)^{2} d u d z d y-4 \int_{0}^{x} m(y) \int_{0}^{y} \int_{0}^{z} m(u) d u d z d y \\
= & x \varphi^{\prime \prime}(x, 0)+4 \int_{0}^{x} \int_{0}^{y} \int_{0}^{z} m(u)^{2} d u d z d y-4 \int_{0}^{x} m(y) \int_{0}^{y} \int_{0}^{z} m(u) d u d z d y .
\end{aligned}
$$

Here we used (6.5). We get the conclusion.

LEMMA 10. Let $l_{1}=-\infty$ and $m((-\infty)+)>-\infty$. Assume that

$$
m(-x)-m((-\infty)+) \sim x^{(\alpha+1)^{-1}-1} K(x) \quad \text { as } \quad x \rightarrow \infty,
$$

where $K(x)$ is slowly varying at $\infty$ and $n<\alpha<n+1$ with $n \geqq 2$. Then $\lambda h(\lambda)$ is $n$ times differentiable in a right neighborhood of 0 ,

$$
\begin{gathered}
\left(h^{\prime} h^{-2}\right)(\lambda) \longrightarrow-\left(c_{0}\right)^{-1}, \\
{\left[h^{-3}\left\{h h^{\prime \prime}-2\left(h^{\prime}\right)^{2}\right\}\right](\lambda) \longrightarrow 2 c_{0}^{-2} c_{1}}
\end{gathered}
$$

$$
\left[h^{-4}\left\{6 h h^{\prime} h^{\prime \prime}-6\left(h^{\prime}\right)^{3}-h^{2} h^{(3)}\right\}\right](\lambda) \longrightarrow 6\left(c_{0}\right)^{-3}\left(c_{1}^{2}-c_{0} c_{2}\right)
$$

as $\lambda \downarrow 0$, where $c_{k}=\lim _{\lambda \downarrow 0} \frac{1}{k !} \cdot \frac{d^{k}}{d \lambda^{k}}\{\lambda h(\lambda)\}$ for $k=0,1$ and 2 .

Proof. Under the assumption of the lemma, it is shown in the proof of Theorem 6 in [10] that $\lambda h(\lambda)$ is $n$ times differentiable in a right neighborhood of 0 and

$$
h(\lambda)=\sum_{k=0}^{n} c_{k} \lambda^{k-1}+(-1)^{n}(1+o(1)) \lambda^{\alpha-1} N(\lambda),
$$

where $N(\lambda)$ is slowly varying at 0 . Since $h(\lambda)$ is completely monotone,

$$
\begin{aligned}
h(\lambda) & =c_{0} \lambda^{-1}+c_{1}+c_{2} \lambda+o(\lambda), \\
h^{\prime}(\lambda) & =-c_{0} \lambda^{-2}+c_{2}+o(1), \\
h^{\prime \prime}(\lambda) & =2 c_{0} \lambda^{-3}+o\left(\lambda^{-1}\right)
\end{aligned}
$$

and

$$
h^{(3)}(\lambda)=-6 c_{0} \lambda^{-4}+o\left(\lambda^{-2}\right)
$$


Hence, we have (6.6). Since

$$
h h^{\prime \prime}(\lambda)=2\left(c_{0}\right)^{2} \lambda^{-4}+2 c_{0} c_{1} \lambda^{-3}+o\left(\lambda^{-2}\right)
$$

and

$$
2\left(h^{\prime}\right)^{2}(\lambda)=2\left(c_{0}\right)^{2} \lambda^{-4}-4 c_{0} c_{2} \lambda^{-2}+o\left(\lambda^{-2}\right),
$$

as $\lambda \downarrow 0$, we have

$$
\left\{h h^{\prime \prime}-2\left(h^{\prime}\right)^{2}\right\}(\lambda)=2 c_{0} c_{1} \lambda^{-3}+O\left(\lambda^{-2}\right) .
$$

Hence we get (6.7). Since

$$
\begin{aligned}
& \left\{h^{(3)} h^{2}\right\}(\lambda)=-6 c_{0}^{3} \lambda^{-6}-12 c_{0}^{2} c_{1} \lambda^{-5}-6 c_{0}\left(c_{1}^{2}+2 c_{0} c_{2}\right) \lambda^{-4}+o\left(\lambda^{-4}\right) \\
& \left\{h^{\prime \prime} h^{\prime} h\right\}(\lambda)=-2 c_{0}^{3} \lambda^{-6}-2 c_{0}^{2} c_{1} \lambda^{-5}+o\left(\lambda^{-4}\right)
\end{aligned}
$$

and

$$
\left(h^{\prime}\right)^{3}(\lambda)=-c_{0}^{3} \lambda^{-6}+3 c_{0}^{2} c_{2} \lambda^{-4}+o\left(\lambda^{-4}\right)
$$

as $\lambda \downarrow 0$, we have

$$
\begin{aligned}
{\left[6 h h^{\prime} h^{\prime \prime}-6\left(h^{\prime}\right)^{3}-h^{2} h^{(3)}\right](\lambda) } & =\left\{6 c_{0}\left(c_{1}^{2}+2 c_{0} c_{2}\right)-18 c_{0}^{2} c_{2}\right\} \lambda^{-4}+o\left(\lambda^{-4}\right) \\
& =6 c_{0}\left(c_{1}^{2}-c_{0} c_{2}\right) \lambda^{-4}+o\left(\lambda^{-4}\right)
\end{aligned}
$$

as $\lambda \downarrow 0$. Hence we get (6.8).

LEMMA 11. Let $u(x, \lambda)=\varphi(x, \lambda)+h(\lambda)^{-1} \phi(x, \lambda)$. Under the assumption of Lemma 10,

$$
\begin{aligned}
& \left\{\left(u^{\prime}\right)^{2}-u^{\prime \prime}\right\}(x, 0) \\
= & \left\{\left(\varphi^{\prime}\right)^{2}-\varphi^{\prime \prime}+2\left(c_{0}\right)^{-1}\left(\phi \varphi^{\prime}-\phi^{\prime}\right)+\left(c_{0}\right)^{-2} \phi\left(\phi+2 c_{1}\right)\right\}(x, 0)
\end{aligned}
$$

and

$$
\begin{aligned}
& \left\{u^{(3)}-3 u^{\prime} u^{\prime \prime}+2\left(u^{\prime}\right)^{3}\right\}(x, 0) \\
= & {\left[\left(\varphi^{(3)}-3 \varphi^{\prime} \varphi^{\prime \prime}+2 \varphi^{3}\right)+3\left(c_{0}\right)^{-1}\left\{\phi^{\prime \prime}-\phi \varphi^{\prime \prime}+2 \varphi^{\prime}\left(\phi \varphi^{\prime}-\phi^{\prime}\right)\right\}\right.} \\
& +6\left(c_{0}\right)^{-2}\left(c_{1}+\phi\right)\left(\phi \varphi^{\prime}-\phi^{\prime}\right) \\
& \left.+2\left(c_{0}\right)^{-3} \phi\left(\phi^{2}+3 c_{1} \phi+3 c_{1}^{2}-3 c_{0} c_{2}\right)\right](x, 0),
\end{aligned}
$$

where $c_{k}$ for $k=0,1,2$ are those defined in Lemma 10. 
Proof. Since $h(0)=\infty, u(x, 0)=\varphi(x, 0)=1$. Since $u^{\prime}=\varphi^{\prime}+\phi^{\prime} h^{-1}$ $\phi^{\prime} h^{-1}-\phi h^{\prime} h^{-2}$, by Lemma 10 ,

$$
\left.h^{\prime}(x, 0)=\left\{\varphi^{\prime}+\left(c_{0}\right)^{-1} \phi\right)\right\}(x, 0) .
$$

Since

$$
u^{\prime \prime}=\varphi^{\prime \prime}+\phi^{\prime \prime} h^{-1}-2 \phi^{\prime} h^{\prime} h^{-2}-\phi h^{-3}\left(h h^{\prime \prime}-2\left(h^{\prime}\right)^{2}\right),
$$

we have, by Lemma 10 ,

$$
u^{\prime \prime}(x, 0)=\left\{\varphi^{\prime \prime}+2\left(c_{0}\right)^{-1} \phi^{\prime}-2 c_{1}\left(c_{0}\right)^{-2} \phi\right\}(x, 0) .
$$

Hence, we have (6.9). Since

$$
\begin{aligned}
u^{(3)}=\varphi^{(3)} & +\phi^{(3)} h^{-1}-3 \phi^{\prime \prime} h^{\prime} h^{-2}-3 \phi^{\prime} h^{-3}\left\{h^{\prime \prime} h-2\left(h^{\prime}\right)^{2}\right\} \\
& +\phi h^{-4}\left\{6 h h^{\prime} h^{\prime \prime}-6\left(h^{\prime}\right)^{3}-h^{2} h^{(3)}\right\},
\end{aligned}
$$

we have, by Lemma 10,

$$
u^{(3)}(x, 0)=\left\{\varphi^{(3)}+3\left(c_{0}\right)^{-1} \phi^{\prime \prime}-6 c_{1}\left(c_{0}\right)^{-2} \phi^{\prime}+6\left(c_{1}^{2}-c_{0} c_{2}\right)\left(c_{0}\right)^{-3} \phi\right\}(x, 0) .
$$

Hence, we have (6.10).

Theorem 9. Let $l_{1}=-\infty$ and $m((-\infty)+)>-\infty$. Let

$$
m(-x)-m((-\infty)+) \sim x^{(\alpha+1)^{-1}-1} K(x) \quad \text { as } \quad x \rightarrow \infty
$$

where $K(x)$ is slowly varying at $\infty$ and $n<\alpha<n+1$ with $n \geqq 2$. If (6.1) holds, then the distribution of $\tilde{\tau}_{x}=\left(\tau_{x}-E_{0} \tau_{x}\right) /\left(V_{0} \tau_{x}\right)^{1 / 2}$ converges to $N(0,1)$ as $x \rightarrow l_{2}$.

Proof. Note that (6.1) implies that $l_{2}<\infty$ or $m\left(l_{2}-\right)=\infty$. Then

$$
\int_{0}^{x} \int_{0}^{y} m(z) d z d y=o\left(\int_{0}^{x} \int_{0}^{y} m(z)^{2} d z d y\right) .
$$

By (4.2), Lemma 9, (6.9) and (6.11), we have

$$
V_{0}\left(\tau_{x}\right) \sim 2 \int_{0}^{x} \int_{0}^{y} m(z)^{2} d z d y \quad \text { as } \quad x \rightarrow l_{2} .
$$

By Lemma 11,

$$
\begin{aligned}
E_{0}\left(\left(\tau_{x}-E_{0}\left(\tau_{x}\right)\right)^{3}\right) & =\left[u^{(3)}-3 u^{\prime \prime} u^{\prime}+2\left(u^{\prime}\right)^{3}\right](x, 0) \\
& =J_{1}(x)+J_{2}(x)+J_{3}(x)+J_{4}(x),
\end{aligned}
$$


where

$$
\begin{aligned}
& J_{1}(x)=\left\{\varphi^{(3)}-3 \varphi^{\prime \prime} \varphi^{\prime}+2\left(\varphi^{\prime}\right)^{3}\right\}(x, 0), \\
& J_{2}(x)=3\left(c_{0}\right)^{-1}\left\{\phi^{\prime \prime}-\phi \varphi^{\prime \prime}+2 \varphi^{\prime}\left(\phi \varphi^{\prime}-\phi^{\prime}\right)\right\}(x, 0), \\
& J_{3}(x)=\left\{6\left(c_{0}\right)^{-2}\left(c_{1}+\phi\right)\left(\phi \varphi^{\prime}-\phi^{\prime}\right)\right\}(x, 0),
\end{aligned}
$$

and

$$
J_{4}(x)=\left\{2\left(c_{0}\right)^{-3} \phi\left(\phi^{2}+3 c_{1} \phi+3 c_{1}^{2}-3 c_{0} c_{2}\right)\right\}(x, 0) .
$$

By the proof of Theorem 2 and by the assumption,

$$
J_{1}(x)=o\left(\left\{\int_{0}^{x} \int_{0}^{y} m(z)^{2} d z\right\}^{3 / 2}\right) \quad \text { as } \quad x \rightarrow l_{2} .
$$

By Lemma 9 and (6.11),

$$
J_{3}(x)=o\left(\left\{\int_{0}^{x} \int_{0}^{y} m(z)^{2} d z\right\}^{3 / 2}\right) \quad \text { as } \quad x \rightarrow l_{2},
$$

while $J_{4}(x)$ is bounded in $0 \leqq x<l_{2}$. By Lemma 9 ,

$$
\begin{aligned}
& c_{0} J_{2}(x) / 3 \\
& =-4\left(\int_{0}^{x} m(y) d y\right)\left(\int_{0}^{x} \int_{0}^{z} m(u) d u d z\right) \\
& \quad+4 \int_{0}^{x}\left(\int_{0}^{z} m(u) d u\right)^{2} d z+4 \int_{0}^{x} \int_{0}^{y} \int_{0}^{z} m(u)^{2} d u d z d y \\
& \quad+4\left(\int_{0}^{x} m(y) d y\right) \int_{0}^{x} \int_{0}^{y} m(z) d z d y \\
& =4 \int_{0}^{x}\left(\int_{0}^{z} m(u) d u\right)^{2} d z+4 \int_{0}^{x} \int_{0}^{y} \int_{0}^{z} m(u)^{2} d u d z d y .
\end{aligned}
$$

Since

$$
\begin{aligned}
& \int_{0}^{x}\left(\int_{0}^{z} m(u) d u\right)^{2} d z=2 \int_{0}^{x} \int_{0}^{y} m(z) \int_{0}^{z} m(u) d u d z d y \\
\leqq & 2 \int_{0}^{x} \int_{0}^{y} m(z) \int_{0}^{z} m(u) d u d z d y,
\end{aligned}
$$

and

$$
\int_{0}^{x} \int_{0}^{y} \int_{0}^{z} m(u)^{2} d u d z d y \leqq \int_{0}^{x} \int_{0}^{y} m(z) \int_{0}^{z} m(u) d u d z d y
$$


we have

$$
J_{2}(x)=o\left(\left\{\int_{0}^{x} \int_{0}^{y} m(z)^{2} d z\right\}^{3 / 2}\right) \quad \text { as } \quad x \rightarrow l_{2}
$$

Hence,

$$
E_{0}\left(\left(\tau_{x}-E_{0}\left(\tau_{x}\right)\right)^{3}\right)=o\left(V_{0}\left(\tau_{x}\right)^{3 / 2}\right) \quad \text { as } \quad x \rightarrow l_{2}
$$

and we get the conclusion by [2] Corollary 2 .

\section{$\S 7$. Comparison with the result of Keller-Kersting-Roesler for diffusion processes}

In this section, we apply our results to diffusion processes and compare them with the result of Keller-Kersting-Roesler. Let $-\infty \leqq r_{1}<r_{2} \leqq \infty$. Let $S$ be either $\left(r_{1}, r_{2}\right),\left[r_{1}, r_{2}\right),\left(r_{1}, r_{2}\right]$ or $\left[r_{1}, r_{2}\right]$. Let $a(y)$ and $b(y)$ be continuous functions on $S$ such that $a(y)>0$ on $\left(r_{1}, r_{2}\right)$. We assume that $0 \in S$. Let $A=a(y) \frac{d^{2}}{d y^{2}}+b(y) \frac{d}{d y}$. We set

$$
\begin{aligned}
& s^{\prime}(y)=\exp \left(-\int_{0}^{y} \frac{b(u)}{a(u)} d u\right), \quad s(x)=\int_{0}^{x} s^{\prime}(y) d y \\
& m^{\prime}(y)=\left(a(y) s^{\prime}(y)\right)^{-1}, \quad m(x)=\int_{0}^{x} m^{\prime}(y) d y
\end{aligned}
$$

Let

$$
\begin{aligned}
& \alpha=\int_{\left(0, r_{2}\right)} s(x) m(d x), \\
& \beta=\int_{\left(0, r_{2}\right)} m(x) s(d x) .
\end{aligned}
$$

If the boundary $r_{2}$ is regular $(\alpha<\infty, \beta<\infty)$ and $r_{2} \in S$, then we need the following boundary condition to determine a process by the differential operator $A$ :

$$
\gamma u\left(r_{2}\right)+\delta A u\left(r_{2}\right)+\mu u^{-}\left(r_{2}\right)=0, \quad \gamma, \delta \leqq 0, \quad \mu \geqq 0, \quad|\delta|+\mu>0
$$

Here $u^{-}\left(r_{2}\right)=\lim _{\varepsilon \downarrow 0} \frac{u\left(r_{2}\right)-u\left(r_{2}-\varepsilon\right)}{s\left(r_{2}\right)-s\left(r_{2}-\varepsilon\right)}$. It is easy to see that $s\left(r_{2}-\right)<\infty$ and $m\left(r_{2}-\right)<\infty$ if and only if $\alpha<\infty$ and $\beta<\infty$. That is, $r_{2}$ is regular if and only if $s\left(r_{2}-\right)+m\left(r_{2}-\right)<\infty$. 
(i) In case $s\left(r_{2}-\right)+m\left(r_{2}-\right)=\infty$, we set $l_{2}=s\left(r_{2}-\right)$ and define $m_{2}(x)$ by

$$
m_{2}(x)= \begin{cases}m\left(s^{-1}(x)\right), & x<l_{2}, \\ \infty, & x>l_{2} .\end{cases}
$$

(ii) In case $s\left(r_{2}-\right)+m\left(r_{2}-\right)<\infty, r_{2} \in S$ and $r_{2}$ is not a trap $(\mu>0$ in $(7.1))$, we let $l_{2}=s\left(r_{2}-\right)+\left|\frac{\mu}{\gamma}\right|$ and define $m_{2}$ by

$$
m_{2}(x)= \begin{cases}m\left(s^{-1}(x)\right), & 0 \leqq x<s\left(r_{2}-\right) \\ m\left(r_{2}-\right)+\left|\frac{\delta}{\mu}\right|, & s\left(r_{2}-\right) \leqq x<l_{2} \\ \infty & l_{2} \leqq x\end{cases}
$$

(iii) In case $s\left(r_{2}-\right)+m\left(r_{2}-\right)<\infty, r_{2} \notin S(\delta=\mu=0$ in (7.1)), we set $l_{2}=s\left(r_{2}-\right)$ and define $m_{2}$ by

$$
m_{2}(x)= \begin{cases}m\left(s^{-1}(x)\right), & x<l_{2}, \\ \infty, & x \geqq l_{2} .\end{cases}
$$

We define $m_{1}$ for $x<0$ in the same way. Let

$$
\tilde{m}(x)= \begin{cases}m_{1}(x), & x<0, \\ m_{2}(x), & x>0 .\end{cases}
$$

Let $\left\{B_{t}\right\}$ be 1-dimensional Brownian motion and let

$$
\phi(t)=\int l(t, y) \tilde{m}(d y) .
$$

Then $\left\{X_{t}=B\left(\phi^{-1}(t)\right)\right\}$ is a generalized diffusion process corresponding to $\tilde{m}$ and $\left\{Y_{t}=s^{-1}\left(X_{t}\right)\right\}$ is the diffusion process with generator $A$ with relevant boundary condition (refer to [6]).

In the following, we assume that

(7.2) $r_{1}=0$ is a reflecting boundary, $a(x)=1$ and $b(x)>0$ for $x>0$.

Let $\sigma_{x}$ be the hitting time of $x$ for $X_{t}$ and let $\tau_{y}$ be the hitting time of $y$ for $Y_{t}$. Then, for $0 \leqq y<r_{2}$,

$$
\begin{aligned}
E_{0}\left(\tau_{y}\right)=E_{0}\left(\sigma_{s(y)}\right) & =\int_{0}^{s(y)} \tilde{m}(u) d u=\int_{0}^{y} m(v) s^{\prime}(v) d v \\
V_{0}\left(\tau_{y}\right)=V_{0}\left(\sigma_{s(y)}\right) & =2 \int_{0}^{s(y)} \int_{0}^{z} \tilde{m}(u)^{2} d u d z \\
& =2 \int_{0}^{y}\left(\int_{0}^{z} m(u)^{2} s^{\prime}(u) d u\right) s^{\prime}(z) d z
\end{aligned}
$$




$$
\begin{aligned}
E_{0}\left(\tau_{y}-E_{0} \tau_{y}\right)^{3} & =E_{0}\left(\sigma_{s(y)}-E_{0} \sigma_{s(y)}\right)^{3} \\
& =12 \int_{0}^{s(y)}\left\{\int_{0}^{z}\left(\tilde{m}(u) \int_{0}^{u} \tilde{m}(v)^{2} d v\right) d u\right\} d z \\
& =12 \int_{0}^{y}\left[\int_{0}^{z}\left\{\tilde{m}(u) \int_{0}^{u} \tilde{m}(v)^{2} s^{\prime}(v) d v s^{\prime}(u)\right\} d u s^{\prime}(z)\right] d z .
\end{aligned}
$$

LEMMA 12. If $r_{2}=\infty$ and

$$
b(x)^{2} \int_{0}^{x} b(y)^{-1} d y \longrightarrow c / 2 \quad \text { as } \quad x \rightarrow \infty,
$$

with $c>0$, then, for any nonnegative integer $n$,

$$
\int_{0}^{x} m^{\prime}(y) b(y)^{-n} d y \sim \frac{c}{c+n+1} m^{\prime}(x) b(x)^{-n-1} \longrightarrow \infty \text { as } x \rightarrow \infty
$$

Proof. By the assumptions (7.2) and (7.3), we have $\int_{0}^{x} m^{\prime}(y) b(y)^{-n} d y$ $\rightarrow \infty$ as $x \rightarrow \infty$ for $n \geqq 0$. Moreover, we have

$$
\begin{aligned}
& \int_{0}^{x} m^{\prime}(y) b(y)^{-n} d y \\
= & \int_{0}^{x} m^{\prime}(y) b(y)^{-n+1} b(y)^{-1} d y \\
\sim & \int_{0}^{x} m^{\prime}(y)\left(\frac{c}{2}\right)^{\frac{-n+1}{2}}\left(\int_{0}^{y} b(z)^{-1} d z\right)^{\frac{n-1}{2}} b(y)^{-1} d y \\
= & m^{\prime}(x)\left(\frac{c}{2}\right)^{\frac{-n+1}{2}}\left(\int_{0}^{x} b(z)^{-1} d z\right)^{\frac{n-1}{2}} \int_{0}^{x} b(z)^{-1} d z \\
& -\int_{0}^{x} m^{\prime \prime}(y)\left(\frac{c}{2}\right)^{\frac{-n+1}{2}}\left(\int_{0}^{y} b(z)^{-1} d z\right)^{\frac{n+1}{2}} d y \\
& -\frac{n-1}{2} \int_{0}^{x} m^{\prime}(y)\left(\frac{c}{2}\right)^{\frac{-n+1}{2}} b(y)^{-1}\left(\int_{0}^{y} b(z)^{-1} d z\right)^{\frac{n-1}{2}} d y \\
& \sim \frac{c}{2} m^{\prime}(x) b(x)^{-n-1}-\frac{c+n-1}{2} \int_{0}^{x} m^{\prime}(y) b(y)^{-n} d y .
\end{aligned}
$$

Here we used the assumption, integration by parts and L'hospital's rule. Hence, we get (7.4).

Under the assumption of Lemma 12,

$$
m(x)=\int_{0}^{x} m^{\prime}(y) d y \sim \frac{c}{c+1} m^{\prime}(x) b(x)^{-1} .
$$


Hence,

$$
\begin{gathered}
\int_{0}^{x} \frac{m(y)}{m^{\prime}(y)} d y \sim \frac{c}{c+1} \int_{0}^{x} b(y)^{-1} d y \sim \frac{c}{2(c+1)} b(x)^{-2} \\
\int_{0}^{x} \frac{m(y)^{2}}{m^{\prime}(y)} d y \sim\left(\frac{c}{c+1}\right)^{2} \int_{0}^{x} m^{\prime}(y) b(y)^{-2} d y \sim \frac{c^{3}}{(c+1)^{2}(c+3)} m^{\prime}(x) b(x)^{-3} .
\end{gathered}
$$

These asymptotics yield

$$
m(x) \int_{0}^{x} \frac{m(y)}{m^{\prime}(y)} d y / \int_{0}^{x} \frac{m(y)^{2}}{m^{\prime}(y)} d y \sim \frac{c+3}{2}
$$

Hence, by Theorem 1, we get the following:

THEOREM 10. Assume (7.2), $r_{2}=\infty$ and (7.3). Then the Laplace transform of the limit distribution of $\tau_{x} / E_{0} \tau_{x}$ as $x \rightarrow \infty$ is $1 / \Phi_{\frac{c+1}{2}}\left(\frac{c+1}{2} \lambda\right)$.

It is shown in [5], that if $b(x)$ is continuously differentiable,

$$
\int^{\infty} b(x)^{-3} d x=\infty
$$

and

$$
b^{\prime}(x)=o\left(b^{2}(x)\right)
$$

then the distribution of $\left\{\tau_{x}-E_{0} \tau_{x}\right\} /\left\{V_{0} \tau_{x}\right\}^{1 / 2}$ converges to $N(0,1)$. This result can also be obtained by using Corollary 2 of Theorem 8 . In order to get this result, the following asymptotic similar to (7.4) is important:

$$
\int_{0}^{x} m^{\prime}(y) b(y)^{-n} d y \sim m^{\prime}(x) b(x)^{-n-1} \longrightarrow \infty \quad \text { as } x \rightarrow \infty \quad \text { for } n \geqq 0 .
$$

We remark that if $b(x) \sim c x^{-1}$ with $c>0$ as $x \rightarrow \infty$, then (7.3) is satisfied; if $b(x) \sim c x^{\alpha}$ with $-1<\alpha<1 / 3$ and $c>0$, then (7.5) and (7.6) are satisfied.

\section{REFERENCES}

[1] L. Bondesson, Class of infinitely divisible distributions and densities, Z. Wahr., 57 (1981), 39-71.

[2] M. Hori and T. Fujimagari, Note on the asymptotic normality of a stochastic process with independent increments, Proc. Japan Acad., 47 suppl. II (1971), 989-993. 
[3] K. Ito and H. P. McKean Jr., Diffusion Processes and Their Sample Paths, 2nd printing, Springer, Berlin, Heiderberg, New York, 1974.

[4] Y. Kasahara, Spectral theory of generalized second order differential operators and its applications to Markov processes, Japan. J. Math., 1 (1975), 67-83.

[5] G. Keller, G. Kersting and U. Roesler, On the asymptotic behavior of first passage times for discussions, Probab. Th. Rel. Fields, 77 (1988), 379-395.

[6] S. Kotani, S. Watanabe, Krein's spectral theory of strings and generalized diffusion processes, Lecture Notes in Math., 923 Springer, 1982, 235-259.

[7] K. Sato, Self-similar processes with independent increments, Probab. Th. Rel. Fields, 89 (1991), 285-300.

[8] E. Seneta, Regularly varying functions, Lecture Notes in Math., 508 Springer, 1976.

[9] A. D. Soloviev, Asymptotic distribution of the moment of first crossing of a high level by a birth and death process, Proc. 6th Berkeley Symposium on Math. Statist. and Probab. Vol. 3, 71-86, University of California Press, Berkeley-Los Angeles, 1972.

[10] M. Yamazato, On subclasses of infinitely divisible distributions on $R$ related to hitting time distributions of 1-dimensional generalized diffusion processes, Nagoya Math. J., 127 (1992), 175-200.

[11] - Hitting time distributions of single points for 1-dimensional generalized diffusion processes, Nagoya Math. J., 119 (1990), 143-172.

[12] Characterizations of the class of hitting time distributions of 1-dimensional generalized diffusion processes, Proc. 6th Japan-USSR Symposium Probability Theory and Mathematical Statistics, 422-428, World Scientific, Singapore-New Jersey-London-Hong Kong, 1992.

Matsuyo Tomisaki

Department of Mathematics

Faculty of Science

Nara Women's University

Nara, 630-8506

Japan

tomisaki@cc.nara-wu.ac.jp

Makoto Yamazato

Department of Mathematical Sciences

College of Science

University of the Ryukyus

Nishihara, Okinawa 903-0213

Japan

yamazato@math.u-ryukyu.ac.jp 